\title{
Space Weather Magnetometer Aboard GEO-KOMPSAT-2A
}

\author{
W. Magnes ${ }^{1}$ - O. Hillenmaier ${ }^{2}$ • H.-U. Auster ${ }^{3}$ P. Brown ${ }^{4}$ - S. Kraft ${ }^{5}$ J. Seon ${ }^{6}$. \\ M. Delva ${ }^{1}$ - A. Valavanoglou ${ }^{1}$ - S. Leitner ${ }^{1}$ - D. Fischer ${ }^{1}$ - G. Berghofer ${ }^{1} \cdot$ Y. Narita $^{1}$. \\ F. Plaschke ${ }^{1}$. M. Volwerk ${ }^{1}$. J. Wilfinger ${ }^{1}$. C. Strauch ${ }^{2}$. J. Ludwig ${ }^{2}$. \\ D. Constantinescu ${ }^{3}$ - K.-H. Fornacon ${ }^{3}$ K. Gebauer ${ }^{3}$ - D. Hercik ${ }^{3}$ - I. Richter ${ }^{3}$. \\ J.P. Eastwood ${ }^{4} \cdot$ J.P. Luntama ${ }^{5}$ A. Hilgers ${ }^{5}$ M. Heil ${ }^{5}$ - G.W. Na ${ }^{6}$ C.H. Lee ${ }^{6}$
}

Received: 3 June 2020 / Accepted: 27 September 2020 / Published online: 21 October 2020

(C) The Author(s) 2020

\begin{abstract}
The South Korean meteorological and environmental satellite GEO-KOMPSAT2A (GK-2A) was launched into geostationary orbit at $128.2^{\circ}$ East on 4 December 2018. The space weather observation aboard GK-2A is performed by the Korea Space Environment Monitor. It consists of three particle detectors, a charging monitor and a four-sensor Service Oriented Spacecraft Magnetometer (SOSMAG).

The magnetometer design aims for avoiding strict magnetic cleanliness requirements for the hosting spacecraft and an automated on-board correction of the dynamic stray fields which are generated by the spacecraft. This is achieved through the use of two science grade fluxgate sensors on an approximately one meter long boom and two additional magnetoresistance sensors mounted within the spacecraft body.

This paper describes the instrument design, discusses the ground calibration methods and results, presents the post-launch correction and calibration achievements based on the data which were acquired during the first year in orbit and demonstrates the in-flight performance of SOSMAG with two science cases.

The dynamic stray fields from the GK-2A spacecraft, which was built without specific magnetic cleanliness considerations, are reduced up to a maximum factor of 35 . The magnitude of the largest remnant field from an active spacecraft disturber is $2.0 \mathrm{nT}$. Due to a daily shadowing of the SOSMAG boom, sensor intrinsic offset oscillations with a periodicity up to 60 minutes and peak-to-peak values up to $5 \mathrm{nT}$ remain in the corrected data product.

The comparison of the cleaned SOSMAG data with the Tsyganenko 2004 magnetic field model and the magnetic field data from the Magnetospheric Multiscale mission demon-
\end{abstract}

\section{W. Magnes}

Space Research Institute, Austrian Academy of Sciences, Graz, Austria

2 Magson GmbH, Berlin, Germany

3 Institut für Geophysik und extra-terrestrische Physik, Technische Universität Braunschweig, Braunschweig, Germany

4 Blackett Laboratory, Imperial College London, London, UK

5 European Space Agency, Paris, France

6 School of Space Research, Kyung Hee University, Suwon, Korea 
strates that the offset error is less than the required $5 \mathrm{nT}$ for all three components and that the drift of the offsets over 10 months is less than $7 \mathrm{nT}$.

Future work will include a further reduction of the remaining artefacts in the final data product with the focus on lessening the temperature driven sensor oscillations with an epoch based identification and correction.

Keywords Space weather - Geostationary orbit · Radiation belt $\cdot$ Multi-sensor magnetometer $\cdot$ Fluxgate sensor $\cdot$ Anisotropic magnetic resistance

\section{Introduction}

Space weather science deals with the time varying conditions within the solar system driven by the dynamics of the Sun (Knipp 2011; Moldwin 2008). It directly affects the stream of charged particles released from the upper atmosphere of the Sun which again connects the solar dynamics to the space surrounding the Earth with its magnetosphere, ionosphere, thermosphere and exosphere (e.g., Eastwood et al. 2017a).

Space weather has a nearly permanent influence on space-borne and ground-based assets (e.g., Eastwood et al. 2017b). To give just a few examples, it causes spacecraft failures through radiation damage and spacecraft charging, affects the level of friction between spacecraft's surfaces and the outer layer of the Earth's atmosphere in low Earth orbits, modulates the radiation environment for humans in space, generates ground induced electric currents and disturbs spacecraft as well as long-distance radio signals.

Forecast as well as nowcast of space weather driven events based on magnetic field measurements (amongst others) is therefore an important service for our modern society with its advanced technological systems (Eastwood et al. 2017b; Hapgood 2012). The measurement of the magnetic field in geostationary orbit at a geocentric distance of 6.6 Earth radii is of specific importance since it can be the first indication that significant space weather has reached Earth (Singer et al. 1996).

GEO-KOMPSAT-2A (GK-2A) is a South Korean meteorological and environmental satellite in geostationary orbit at a geographic longitude of $128.2^{\circ}$ East. GK-2A is managed by the Korea Meteorological Administration, and it was built by the Korea Aerospace Research Institute (KARI). The satellite hosts the Korea Space Environment Monitor (KSEM) instrument suite, which was developed under the lead of the Kyung Hee University. It consists of three particle detectors, a charging monitor and a four-sensor Service Oriented Spacecraft Magnetometer (SOSMAG).

The functional composition of the KSEM instrument suite is illustrated in Fig. 1. A dedicated Instrument Data Processing Unit (IDPU) is allocated for the three particle detectors (Seon et al. 2020) and the charging monitor to handle scientific data, telemetry and spacecraft command, whereas another independent Data Processing Unit within the SOSMAG electronics box handles data, telemetry and commands for the four-sensor magnetometer. All SOSMAG commands and data are routed via the IDPU. The SOSMAG development was initiated and conducted by the European Space Agency and built by the SOSMAG consortium: Magson GmbH, Austrian Academy of Sciences, Technische Universität Braunschweig and Imperial College London.

The instrument has been designed as a ready-to-use space magnetometer with multiple magnetic field sensors, a short deployable boom, a large magnetic field range to cover full Earth's field in low Earth orbit, a low noise floor, a suitable radiation hardness for orbits crossing the radiation belts and a flexible digital as well as power interfaces. The instrument 


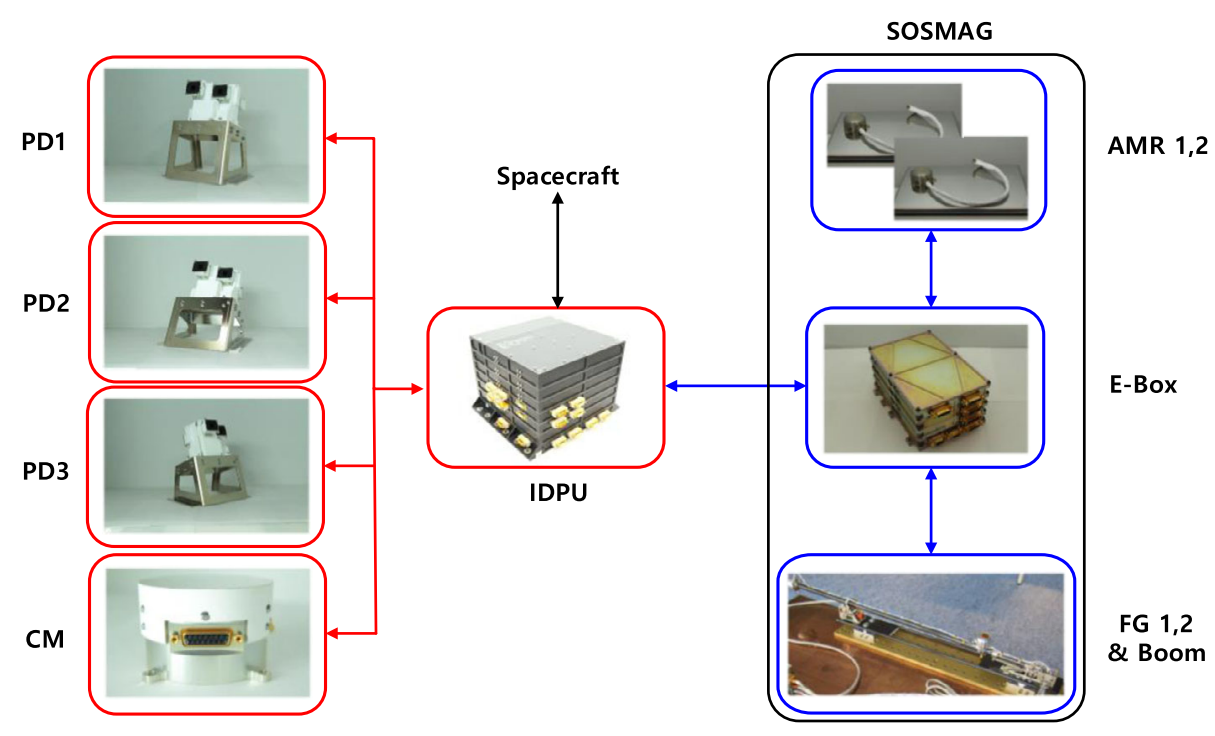

Fig. 1 Configuration of the Korea Space Environment Monitor (KSEM). KSEM consists of three Particle Detectors (PDs), a Charging Monitor (CM) and the Service Oriented Spacecraft Magnetometer (SOSMAG) (figure from Seon et al. 2020)

concept shall enable suitable measurements of the natural magnetic field in space aboard spacecraft which are not directly related to space weather investigations (e.g. meteorological satellites). Such missions are often placed in orbits which are relevant for doing space science in general as well as space weather now- and forecasting in particular.

The combination of multiple sensors and an approximately one meter long boom, which can hold two magnetic field sensors, was chosen to avoid the need of imposing costly magnetic cleanliness requirements onto the hosting spacecraft. The instrument configuration aboard the GK-2A mission contains two science grade fluxgate sensors on the boom and two additional magnetoresistance sensors mounted within the spacecraft body. The two latter sensors are ideally located in the vicinity of dynamic magnetic field sources like reaction wheels, heater elements and magnetic actuators. The measurements of the two spacecraft sensors together with the inner boom sensor enable an automated correction of the dynamic, spacecraft generated disturbances from the measurements of the outer boom sensor. The correction can be done by the on-board software.

The first power-on of SOSMAG including successful boom release took place on 4 January 2019. After the commissioning of all KSEM sensors as well as the primary payload of GK-2A, SOSMAG started regular operation on 25 February 2019 with all four sensors taking data at one vector per second.

In this paper, Sect. 2 discusses the science objectives for the magnetic field measurements aboard GK-2A and the derived performance requirements for the SOSMAG instrument. Section 3 explains the instrument design with a brief description of all main hardware elements while Sect. 4 deals with the on-ground calibration. Section 5 outlines the flight operation with a detailed discussion of the removal of the magnetic contamination from the spacecraft as well as the determination of offsets and alignment of the cleaned SOSMAG data. The description of exemplary science observations complement this section. The data product flow of SOSMAG is explained in Sect. 6, and Sect. 7 summarizes this review. 
Fig. 2 Superposed plot of the local time distribution of the magnetic field magnitude measured by GOES-12 on GEO. Gray points are the data for the year 2007. Red curve is the average value of the data. Green and blue curves are the averages for the summer and winter seasons, respectively. The dashed yellow line indicates the intrinsic field of the Earth. (Figure from Borovsky and Denton 2010)

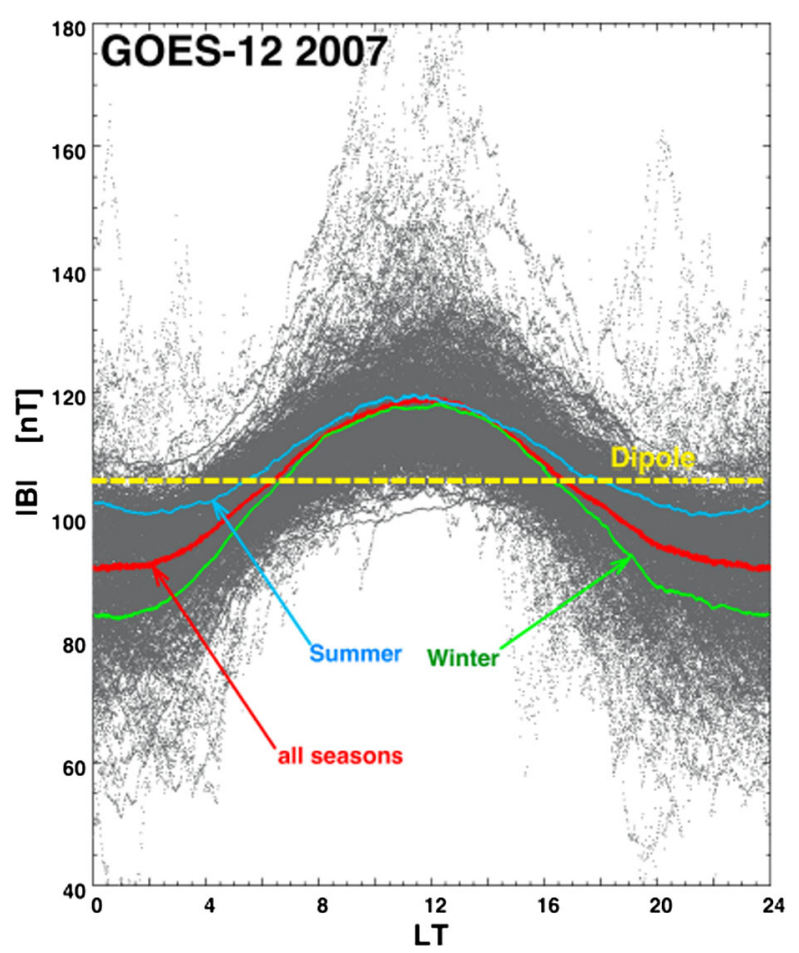

\section{Science Objectives and Performance Requirements}

The circular GEostationary Orbit (GEO) is located at a distance of approximately 42,164 km (or 6.6 Earth radii, RE) from the Earth's centre. Under most conditions, GEO lies within the Earth's outer magnetosphere, as the subsolar standoff distance of the dayside magnetospheric boundary (the magnetopause) is typically on the order of $10 \mathrm{RE}$ (or about $63,500 \mathrm{~km}$ from the Earth's centre). Along GEO, the magnetic field ranges from about $\pm 100 \mathrm{nT}$ to $\pm 2,000 \mathrm{nT}$ (Singer et al. 1996; Pulkkinen et al. 2006). The local time distribution of the magnetic field magnitude at GEO, based on GOES-12 measurements of 2007, is shown in Fig. 2. Under more unusual, specific solar wind conditions, such as high-speed streams and coronal mass ejections, the magnetosphere can be severely compressed due to the intense dynamic and magnetic pressures of the solar wind. Consequently, the magnetopause may sweep over the dayside part of GEO, and spacecraft on that orbit may find themselves located outside the magnetosphere, in the magnetosheath. This is a transition region between the bow shock and the magnetopause where heated and compressed solar wind flows around the magnetospheric obstacle.

Compression yields one option for energy input to the magnetosphere. A more significant energy and particle input occurs, however, by means of magnetic reconnection (Eastwood et al. 2017a). At the dayside magnetopause, reconnection is enhanced when the interplanetary magnetic field (IMF) and the terrestrial magnetic field are anti-parallel, i.e., when the IMF points in southward direction. This leads to energy and magnetic flux input to the magnetospheric tail until tail reconnection occurs. Thereby, particles and energy flow from the tail towards Earth in what is called a magnetospheric substorm.

A southward rotation of the IMF is known to trigger substorms. When a substorm occurs, a sudden decrease can be seen in the northward component of the Earth's magnetic field (i.e., 


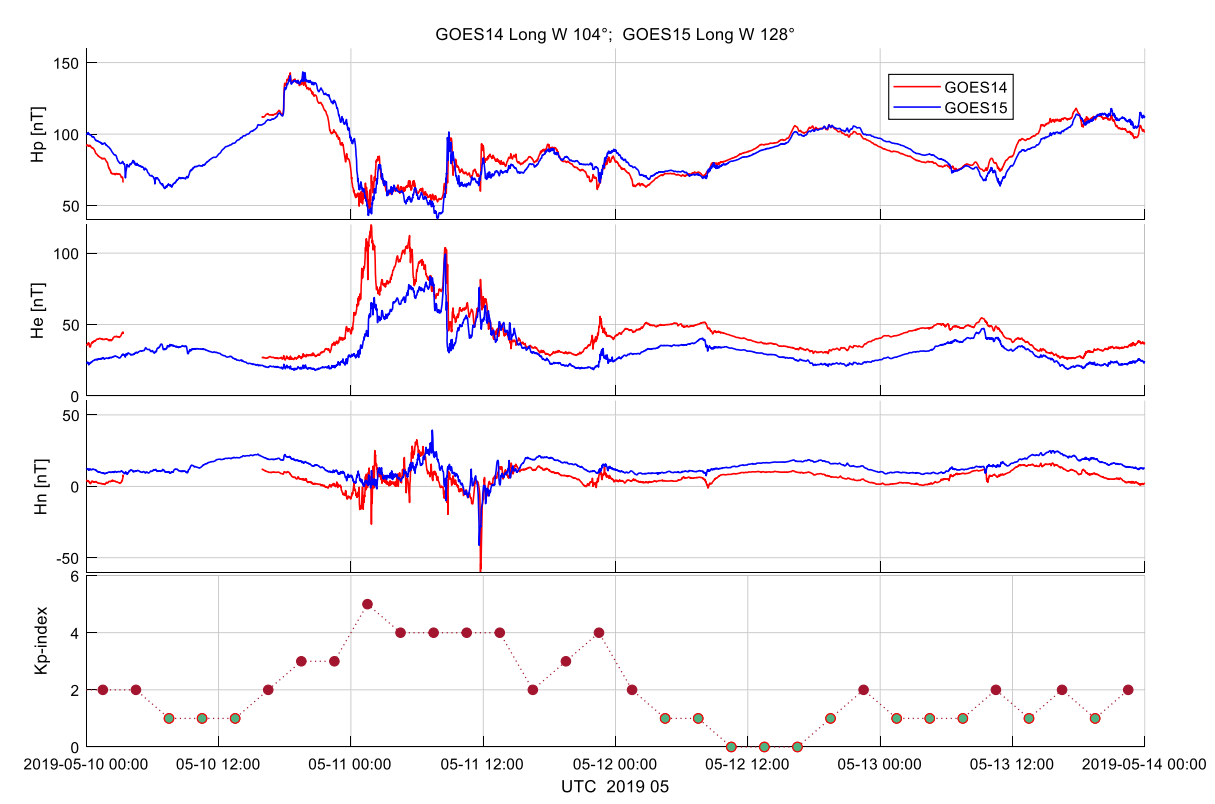

Fig. 3 Four days of magnetic field data obtained by GOES-14 and -15 spacecraft with magnetic activity on 11 May 2019 (data from NOAA Space Weather Prediction Center; http://www.swpc.noaa.gov/)

the component parallel to the Earth's rotation axis) in the magnetotail, reaching nearly zero or even negative values at GEO. This can be in principle be used as a detection method for substorms.

Magnetotail reconnection can even occur as close to Earth as 6.6-10 RE. As shown recently, near-Earth reconnection powered an intense magnetic storm (Angelopoulos et al. 2020), supporting the importance of space weather measurements in GEO.

Figure 3 displays the magnetic field variation measured by the GOES-14 and 15 magnetometers during four days in May 2019 with magnetic activity on 11 May. The parallel component (Hp) recorded by GOES-14 (15) is shown in red (blue) in the uppermost plot. This component is perpendicular to the satellite orbital plane and is essentially parallel to Earth's rotation axis. If this component drops to near zero or even below (i.e., field direction change into the anti-parallel direction) whilst on the dayside, the Earth's magnetosphere is likely so compressed that the magnetopause is located within the radius of GEO. If the decrease in the parallel component occurs on the nightside, it is an indication for the presence of strong currents flowing in the magnetotail, likely associated with magnetic substorms.

The magnetic field measurements on a network of GEO satellites that enable

1. the detection of magnetopause crossings for very active geomagnetic storms,

2. the detection of shocks and sudden impulses in the solar wind, and

3. the assessment of the level of geomagnetic activity from related variations of the field,

are therefore essential for monitoring space weather and for supporting, e.g., rocket launch decisions and other real-time activities (Singer et al. 1996). This has led to a number of performance requirements for the final data product (Table 1) and related design requirements for the magnetometer aboard the GK-2A spacecraft (Table 2) during the design phase. Lessons learned in this regard during the first year in operation are discussed in Sect. 7. 
Table 1 Performance requirements for the final data product

\begin{tabular}{ll}
\hline Parameter & Requirement \\
\hline Dynamic range & $\pm 1,000 \mathrm{nT}$ \\
Error $(\text { signal frequency }<0.1 \mathrm{mHz})^{\mathrm{a}}$ & $\left|E_{l}\right|<5 \mathrm{nT}$ per axis \\
Error $(0.1 \mathrm{mHz}<\text { signal frequency }<64 \mathrm{~Hz})^{\mathrm{b}}$ & $\left|E_{h}\right|<1 \mathrm{nT}$ per axis \\
Precision & $<15 \mathrm{pTHz}^{-0.5}$ at $1 \mathrm{~Hz}$ \\
Life time & 10 years \\
Knowledge of alignment & $\left|E_{a}\right|<10$ degrees \\
Vector rate & $\geq 1 \mathrm{~Hz}$ \\
\hline
\end{tabular}

${ }^{\text {a } F r e q u e n c y ~ r a n g e ~ r e l e v a n t ~ f o r ~ t h e ~ c l a s s i c a l ~ o f f s e t ~ d e t e r m i n a t i o n ~}$

${ }^{b}$ Important for the accurate measurement of the field variations

Table 2 Design requirements for the SOSMAG magnetic field sensors

\begin{tabular}{|c|c|}
\hline Parameter & Requirement \\
\hline Dynamic range $^{a}$ & $\pm 50,000 \mathrm{nT}$ \\
\hline Amplitude spectral density of boom sensors & $<15 \mathrm{pT} \mathrm{Hz}^{-0.5}$ at $1 \mathrm{~Hz}$ \\
\hline Amplitude spectral density of spacecraft sensors & $<150 \mathrm{pT} \mathrm{Hz}^{-0.5}$ at $1 \mathrm{~Hz}$ \\
\hline Offset stability vs. sensor temp. of the boom sensors ${ }^{b}$ & $<0.1 \mathrm{nT} \mathrm{K}^{-1}$ \\
\hline Offset stability vs. sensor temp. of the spacecraft sensors ${ }^{c}$ & $<3 \mathrm{nTK}^{-1}$ \\
\hline Offset stability vs. electronics temp. of the boom sensors & $<0.05 \mathrm{nT} \mathrm{K}^{-1}$ \\
\hline Offset stability vs. electronics temp. of the spacecraft sensors & $<0.5 \mathrm{nT} \mathrm{K}^{-1}$ \\
\hline Gain stability vs. sensor temp. of the boom sensors ${ }^{\mathrm{d}}$ & $<20 \mathrm{ppm} \mathrm{K}^{-1}$ \\
\hline Gain stability vs. sensor temp. of the spacecraft sensors ${ }^{d}$ & $<70 \mathrm{ppm} \mathrm{K}^{-1}$ \\
\hline Digital resolution & $<0.1 \mathrm{nT}$ \\
\hline Life time & 10 years \\
\hline
\end{tabular}

a To allow for functional tests in full Earth's field on ground and to cover unexpectedly large background fields from the spacecraft

${ }^{\mathrm{b}}$ The offset stability requirement for the boom mounted fluxgate sensors is defined by dividing max. $\left|E_{l}\right|$ of $5 \mathrm{nT}$ in Table 1 by the expected temperature swing which was assumed $50 \mathrm{~K}$ for the fluxgate sensors before launch

${ }^{\mathrm{c}}$ The offset stability requirement for the spacecraft sensors can be relaxed by a factor of 30 because the spacecraft related disturbances are at least by this factor larger at the position of the spacecraft sensors than they are at the position of the outer fluxgate sensor on the boom

${ }^{\mathrm{d}}$ The gain stability requirements are defined by taking max $\left|E_{l}\right|$ (from Table 1), the realistic dynamic range at the sensor positions, a temperature uncertainty of $10 \mathrm{~K}$ and a relaxation factor for the spacecraft sensors into account

\section{Instrument Description}

\subsection{Overview}

SOSMAG is a multi-sensor magnetometer with an approximately one meter long boom. The configuration as built for the GK-2A spacecraft as part of the KSEM instrument suite contains two science grade, boom mounted fluxgate sensors, two anisotropic magnetoresistance 


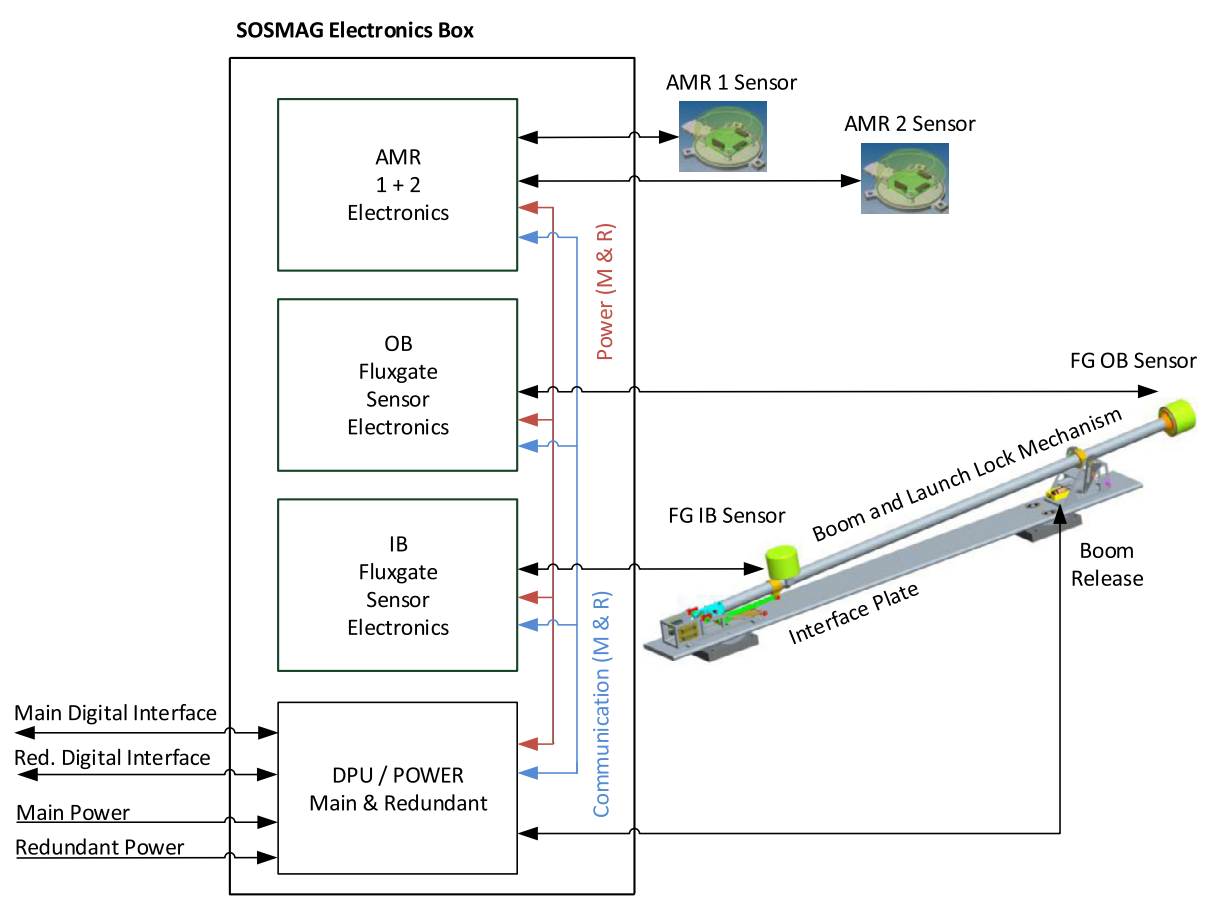

Fig. 4 Block diagram of the SOSMAG instrument (AMR: Anisotropic Magneto-Resistance, DPU: Digital Processing Unit, FG: FluxGate, IB: InBoard, OB: OutBoard)

sensors which are mounted within the spacecraft body, a deployable boom, which includes a specific mounting interface to avoid mismatches of the thermal expansion coefficients, and an electronics box with the data processing, power conversion and sensor front-end electronics.

The block diagram of the SOSMAG instrument is shown in Fig. 4 and the main instrument parameters are listed in Table 3. Pictures of the SOSMAG qualification model and the boom mounted to the $+\mathrm{X}$ sidewall of the spacecraft are depicted in Fig. 5 and Fig. 6, respectively. The modular concept of SOSMAG would allow for one additional sensor front-end electronics board within the electronics box, which connects to either one more fluxgate sensor or two more Anisotropic Magneto-Resistance (AMR) sensors.

SOSMAG measures the magnetic field continuously with a maximum vector rate of 128 vectors per second at all four sensors, and it generates adequate housekeeping information. Within the Data Processing Unit (DPU), the magnetic field data can be filtered and decimated by a commandable integer factor. With e.g. a decimation factor of 128 , it is reduced to 1 vector per second. The filter coefficients are equal to one over the decimation factor. It results in a transfer characteristics which is equivalent to a boxcar filter with rectangular windowing.

In addition, a cleaned vector can be calculated by the SOSMAG DPU. It is a linear combination of the measurements from all sensors. The data from the magnetic sensors are multiplied with a $3 \times 3$ calibration matrix and corrected for a static offset value before the cleaned vector is processed.

All SOSMAG commands and data are routed via the Instrument Data Processing Unit (IDPU) of the KSEM instrument suite (Fig. 1). Also the power switching for SOSMAG is 


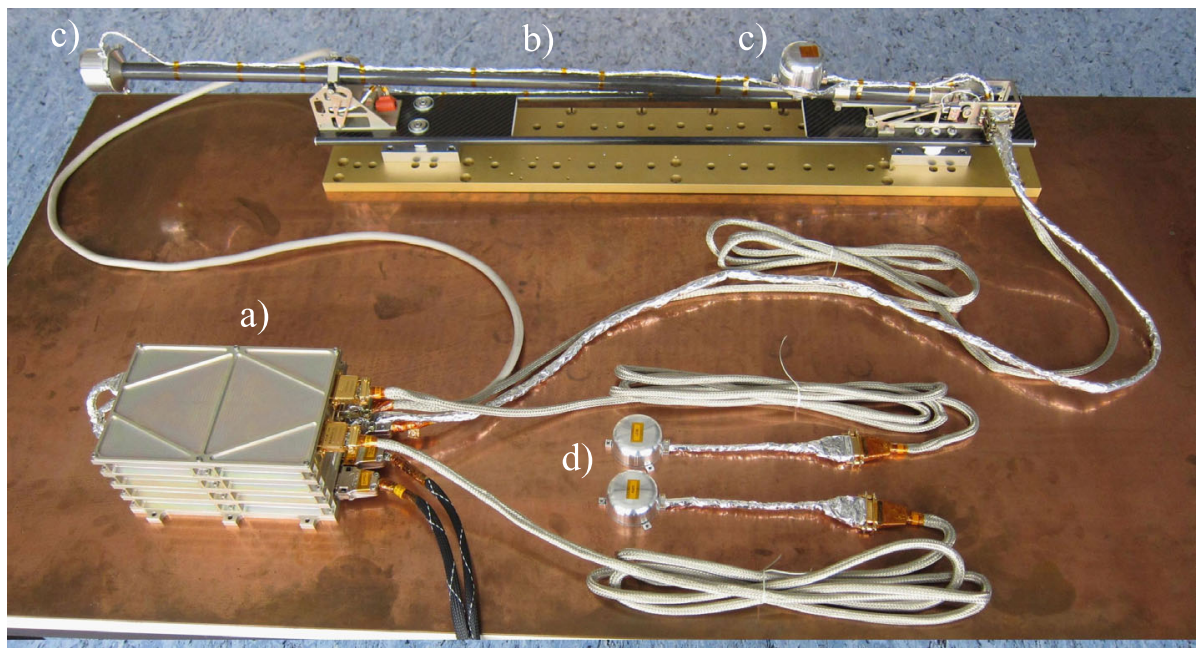

Fig. 5 Picture of the SOSMAG qualification model with (a) electronics box, (b) boom with carbon fibre based interface plate, (c) two fluxgate sensors mounted onto the boom and (d) two anisotropic magnetoresistance sensors

Table 3 Main instrument parameters

${ }^{a}$ Average power consumption from $50 \mathrm{~V}$ at room temperature which includes the power loss in a linear voltage regulator circuit which reduces the voltage from $50 \mathrm{~V}$ to $28 \mathrm{~V}$. The power consumption of SOSMAG from $28 \mathrm{~V}$ is $4.6 \mathrm{~W}$

${ }^{\mathrm{b}}$ The wall thickness was increased to $4 \mathrm{~mm}$ for a better radiation protection in geostationary orbit

${ }^{\mathrm{c}}$ Including sensor harness along the boom but without thermal protection

${ }^{\mathrm{d}}$ The maximum vector rate of 128 vectors per second can be decimated by a commandable integer factor by the DPU (with e.g. 128 it is reduced to 1 vector per second)

\begin{tabular}{|c|c|}
\hline Parameter & Performance \\
\hline Power consumption $^{\mathrm{a}}$ & $8.25 \mathrm{~W}$ \\
\hline \multicolumn{2}{|l|}{ Mass: } \\
\hline Electronics box ${ }^{\mathrm{b}}$ & $3211 \mathrm{~g}$ \\
\hline Boom with two fluxgate sensors ${ }^{\mathrm{c}}$ & $1316 \mathrm{~g}$ \\
\hline AMR sensors 1 and 2 with harness & $366 \mathrm{~g}$ \\
\hline Fluxgate and boom release harness & $589 \mathrm{~g}$ \\
\hline Total & $5482 \mathrm{~g}$ \\
\hline \multicolumn{2}{|l|}{ Envelope dimensions } \\
\hline Electronics box & $170 \times 255 \times 110 \mathrm{~mm}^{3}$ \\
\hline Boom with two fluxgate sensors & $1182 \times 96 \times 147 \mathrm{~mm}^{3}$ \\
\hline Maximum vector rate ${ }^{\mathrm{d}}$ & 128 vectors/second \\
\hline \multicolumn{2}{|l|}{ Noise density at $1 \mathrm{~Hz}$} \\
\hline Fluxgate sensors & $<10 \mathrm{pT} \mathrm{Hz}^{-0.5}$ \\
\hline AMR sensors & $<220 \mathrm{pT} \mathrm{Hz}^{-0.5}$ \\
\hline Range & $\pm 65,000 \mathrm{nT}$ \\
\hline \multicolumn{2}{|l|}{ Quantization resolution } \\
\hline Fluxgate sensors & $7.8 \mathrm{pT}$ \\
\hline AMR sensors & $62 \mathrm{pT}$ \\
\hline
\end{tabular}

included in the IDPU electronics. The data routing through the IDPU implies a bandwidth limitation which reduces the maximum possible vector rates transmitted from the GK-2A spacecraft to ground. All operational modes and their respective vector rates are listed in Ta- 
Fig. 6 GK-2A spacecraft during a pre-launch test campaign. The stowed SOSMAG boom with the two fluxgate sensors is indicated with a dotted rectangle. The approximate mounting position of the two AMR sensors within the spacecraft is shown by two white dots. The AMR 1 sensor is located near the $+\mathrm{X} /+\mathrm{Y}$ corner of the spacecraft and the AMR 2 is mounted close to the $+\mathrm{X}$ side wall next to the launch lock of the boom. This image is provided by the Korea Aerospace Research Institute (KARI) and is publicly available at https://www.kari. re.kr/eng/sub03_02_02.do\#link

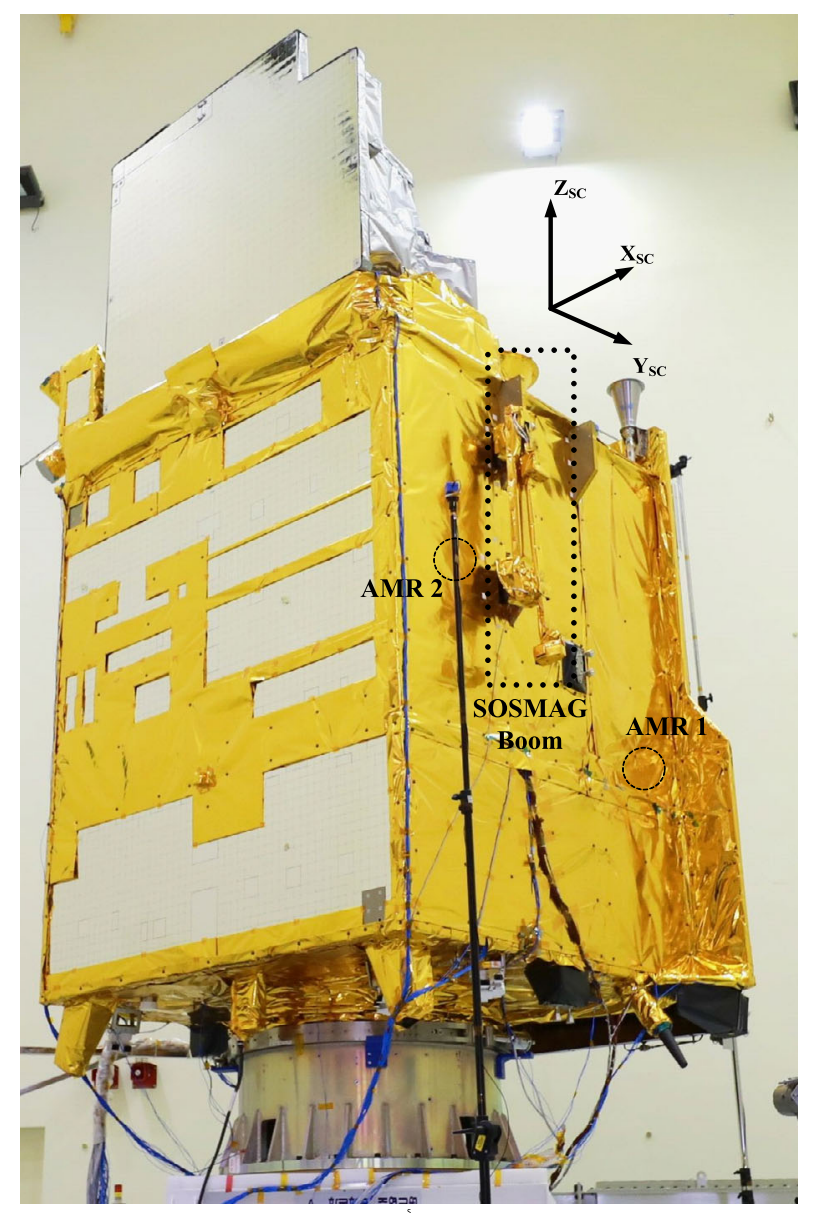

ble 4. They are defined via the number of sensors, the commanded data rate and the optional transmission of the additionally processed cleaned vector. In principle, there is no transmission of the cleaned vector foreseen in the commissioning modes except for one specific mode in which the vector rate of the four magnetic field sensors is reduced to eight. Transmission of the cleaned vector is mandatory for the nominal operational modes of SOSMAG.

The commissioning modes, which can only be enabled when the other KSEM sensors are turned off, are used for instrument check-out and the characterisation of potential dynamic disturbers especially during the early phase of the mission. The two nominal modes, all four sensors plus cleaned vector with one vector per second or just the cleaned vector with 4 vectors per second, are to be run during the science phase.

The data from the first 12 months of nominal operation were studied on ground to characterize the dynamic spacecraft disturbances and to determine correction factors for each specific disturber (see Sect. 5.2). The correction factors can be uploaded to SOSMAG so that an automated correction of these dynamic effects can take place on-board. The cleaned vectors are a linear combination of measurements from all sensors with the coefficients tuned such that the spacecraft disturbance is removed. The two AMR sensors mounted within the spacecraft see in principle larger disturbances and have thus less weight in the corrected 
Table 4 Operational modes of SOSMAG aboard GK-2A

\begin{tabular}{llll}
\hline Mode name & \# Sensors & Cleaned vector & Vector rate [vectors/sec.] \\
\hline Commissioning 1S_64H & 1 & No & 64 \\
Commissioning 1S_32H & 1 & No & 32 \\
Commissioning 2S_32H & 2 & No & 32 \\
Commissioning 2S_16H & 2 & No & 16 \\
Commissioning 4S_16H & 4 & No & 16 \\
Commissioning 4S_8H & 4 & No & 8 \\
Commissioning 5V_8H & 4 & Yes & 8 \\
Commissioning 4S_1H & 4 & No & 1 \\
Normal 1 V & 0 & Yes & 4 \\
Normal 5 V & 4 & Yes & 1 \\
Standby & 0 & DPU on and sensors off & \\
\hline
\end{tabular}

measurements. This is why the accuracy requirements for the spacecraft sensors could be relaxed (Table 2) which made it possible to select AMR sensors for the measurements within the spacecraft.

\subsection{Electronics Box and AMR Sensors}

The SOSMAG electronics is housed in one metal box fabricated from Aluminium. The upper left picture of Fig. 7 shows the flight model of the electronics box with bottom plate, data processing and power distribution frame, two fluxgate front-end electronics frames, AMR front-end electronics frame and lid. The box design allows for a flexible scaling of the number of frames between two and five according to the target application.

The fully redundant processing and power distribution unit is accommodated on a single printed circuit board (upper right picture of Fig. 7). The power distribution section reduces the supply voltage provided by the KSEM IDPU (Fig. 1) from $50 \mathrm{~V}$ to less than $30 \mathrm{~V}$ via a linear voltage regulator and connects it to the instrument internal interface bus. Each of the four electronics boards has its own power converter with an output voltage of $\pm 5 \mathrm{~V}$ (Fig. 4).

The main functionalities of the Data Processing Unit (DPU) are the commanding of the three clients including the enabling of the power converter outputs of each client separately, the measurement of the primary supply currents and voltages, the activation of the release mechanism of the boom, the DPU internal housekeeping measurements, which includes boom related status information, and the processing of the magnetic field vectors from the clients (Sect. 3.1). The raw data from the magnetic sensors, which are received with 128 vectors per second, can be multiplied with a $3 \times 3$ calibration matrix and corrected for a static offset value before the cleaned vector is processed (Sect. 3.1). All required parameters can be uploaded to the instrument from ground. The instrument firmware is compiled to a custom-made processor with a reduced set of instructions (Auster et al. 2008), which runs as a core element in the field programmable gate array.

The front-end electronics of the two fluxgate sensors (Primdahl 1979) features a direct digitization of the amplified voltage from the pick-up coils (Sect. 3.3) at a sampling frequency which is four times the excitation frequency (Auster et al. 1995). Analogue circuitries like filters and phase-sensitive demodulators are replaced by digital signal processing to improve measurement stability. The excitation frequency is $9.6 \mathrm{kHz}$ and digital-toanalogue converters are used to null the field within the centre of the fluxgate sensor via the 

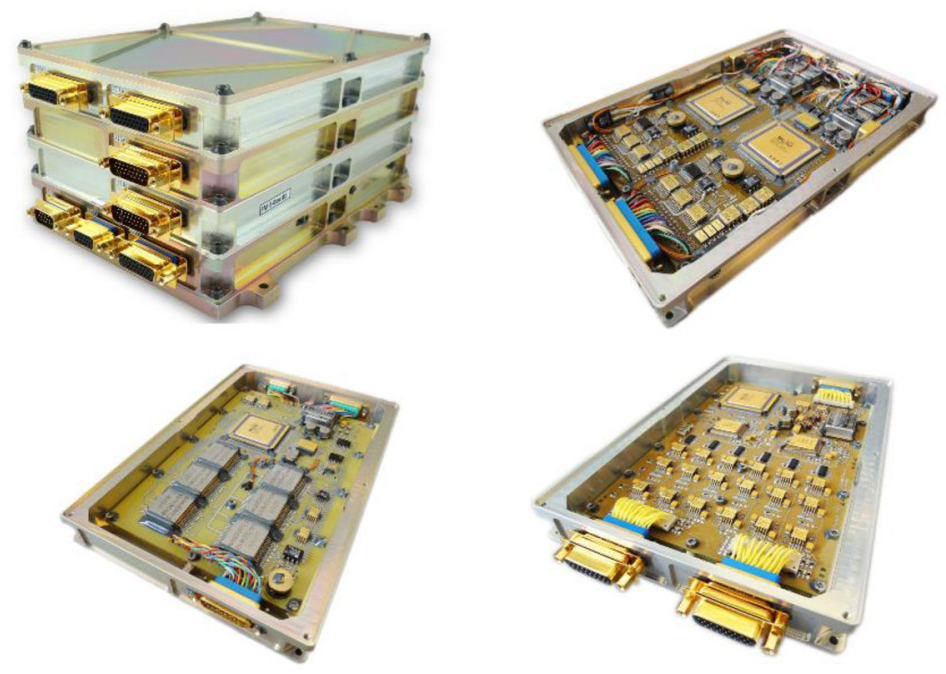

Fig. 7 Pictures of the SOSMAG electronics box (upper left), the combined data processing and power distribution unit (upper right), the front-end electronics for the fluxgate sensors (lower left), which is implemented twice, and the front-end electronics for the two AMR sensors (lower right). The envelope of the electronics box is $170 \times 255 \times 110 \mathrm{~mm}^{3}$

feedback coils within the sensor to increase the overall linearity and stability. The primarily digital electronics design was first developed for the Rosetta Lander magnetometer (Auster et al. 2007) and has already been used for a number of other space missions (Auster et al. 2008).

For SOSMAG, two different hybrid chips are used. The ADC hybrid contains the preamplifier and the analogue-to-digital converter (18 bits, 1 MSPS), and the DAC hybrid is made up from two 16-bit digital-to-analogue converters and a current source for the feedback system. The hybrids are enclosed in a specific package which increases the radiation hardness of the hybrid electronics.

The electronics box and both, DPU and fluxgate electronics, have been developed by Magson $\mathrm{GmbH}$ in Berlin.

For the close-up measurement of the magnetic disturbers on the spacecraft, a dual-sensor magnetometer based on the Anisotropic Magneto-Resistance (AMR) effect (Brown et al. 2012; Lenz and Edelstein 2006) was developed by the Austrian Academy of Sciences in cooperation with Imperial College London. The two sensors are each composed of three Honeywell HMC1021 sensor elements, a temperature sensor and a die of a full-bridge MOSFET driver circuit with one corresponding non-magnetic bypass capacitor (Fig. 8).

The HMC1021, which is supplied with a bridge voltage of $5 \mathrm{~V}$ in this design, had to be selected as sensing element even though it has a higher noise floor than the HMC1001 (Stutzke et al. 2005). The advantage of the HMC1021 is the higher coil constant of the chip internal offset straps which are used as feedback coils in the closed loop design of the AMR magnetometers. With the HMC1001 as well as with a higher bridge voltage it wouldn't have been possible to stay within the $1.5 \mathrm{~W}$ total power consumption limit which is set via the power converter circuit on each of the front-end electronics boards.

The AMR sensors connect to a single front-end electronics board (lower right picture in Fig. 7) which hosts two mixed-signal application-specific integrated circuits as core elements (Magnes et al. 2008), a field programmable gate array and six digital-to-analogue 
Fig. 8 Photo of the triaxial AMR sensor with opened lid. It consists of a ceramics based printed circuit board, three HMC1021 sensor elements, a bypass capacitor, a temperature sensor, a MICREL 4424 die mounted in chip-on-board technology and a small Tantalum plate for the radiation shielding of the die. The circumference of the baseplate has a diameter of $72 \mathrm{~mm}$

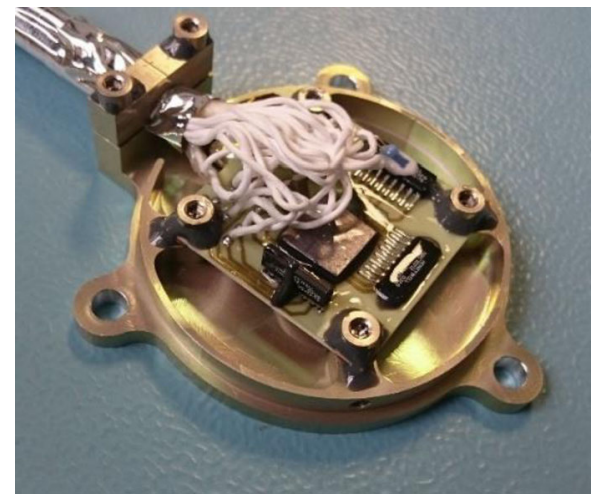

converters with improved Howland current sources. A detailed description of the AMR magnetometer design with its pulsed driver circuit for the periodic magnetization of the ferromagnetic elements in the Honeywell sensor elements can be found in Leitner et al. 2015.

\subsection{Instrument Boom and Fluxgate Sensors}

The SOSMAG boom, which was developed and fabricated by the Technical University Braunschweig, bears the heritage of the Rosetta Lander (Auster et al. 2007; Bibring et al. 2007) and Venus Express magnetometer (Zhang et al. 2006) booms. The main elements of the boom are a one meter long carbon fibre tube, the boom hinge, the launch lock, two fluxgate sensors and a base plate with one fixed and one slightly flexible mounting point to balance out the difference between the thermal expansion coefficients of the carbon fibre base plate and the Aluminium based side panel of the spacecraft to which the boom is mounted to (Fig. 9). The outboard fluxgate sensor is mounted to the boom tip. For boom deployment, the launch lock is opened by breaking the notch of a Nickel-Titanium fastener via a shape memory alloy (i.e. a Frangibolt release system) which is heated up for boom release. In the deployed position the boom is locked by a lever arm (Fig. 10). A number of design updates have been implemented compared to the Venus Express boom. It includes the addition of the inboard fluxgate sensor, the substitution of a pyro element based with a Frangibolt based boom release initiator, the redesign of the boom hinge for increasing the robustness concerning mounting and adjustment tolerances and the inclusion of the additional interface plate.

The inboard fluxgate sensor is also mounted on the boom but closer to the spacecraft, (1) to benefit from the effect of the magnetic field dependence along the (radial) distance from sources on the spacecraft, exactly as initially suggested by Ness et al. (1971), (2) to have it further away from the sources than the spacecraft mounted AMR sensors and (3) to reduce the number of mechanical interfaces with the spacecraft.

The two fluxgate sensors mounted on the boom are nearly identical to the ones of the Rosetta Lander (Auster et al. 2007), Venus Express (Zhang et al. 2006), Themis (Auster et al. 2008) and BepiColombo magnetometers and similar to the ones flown on Equator-S (Fornacon et al. 1999).

The sensor consists of two crossed ring-cores, three pickup coils and a vector compensating feedback coil system, which is used to keep the ring-cores in nearly zero field (Fig. 11). The ring-core elements contain a soft-magnetic and ultra-stable Permalloy band with $13 \%$ 
b)

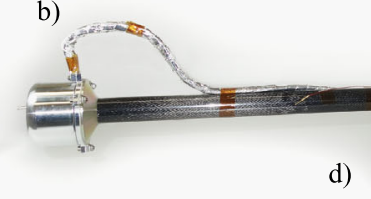

d) c)

a)

e)

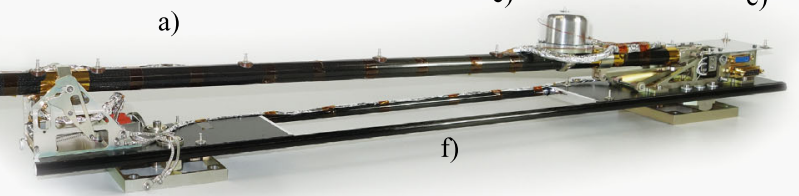

Fig. 9 SOSMAG boom with (a) carbon fibre tube, (b) outboard fluxgate sensor, (c) inboard fluxgate sensor, (d) launch lock with release mechanism, (e) boom hinge and (f) carbon fibre interface plate. In flight configuration, boom and sensors are fully covered with multi-layer insulation. The envelope of the boom is $1182 \times 96 \times 147 \mathrm{~mm}^{3}$

Fig. 10 Drawings of the deployed boom root which is locked via the lever arm (left) and the launch lock with Frangibolt based release mechanism (right)
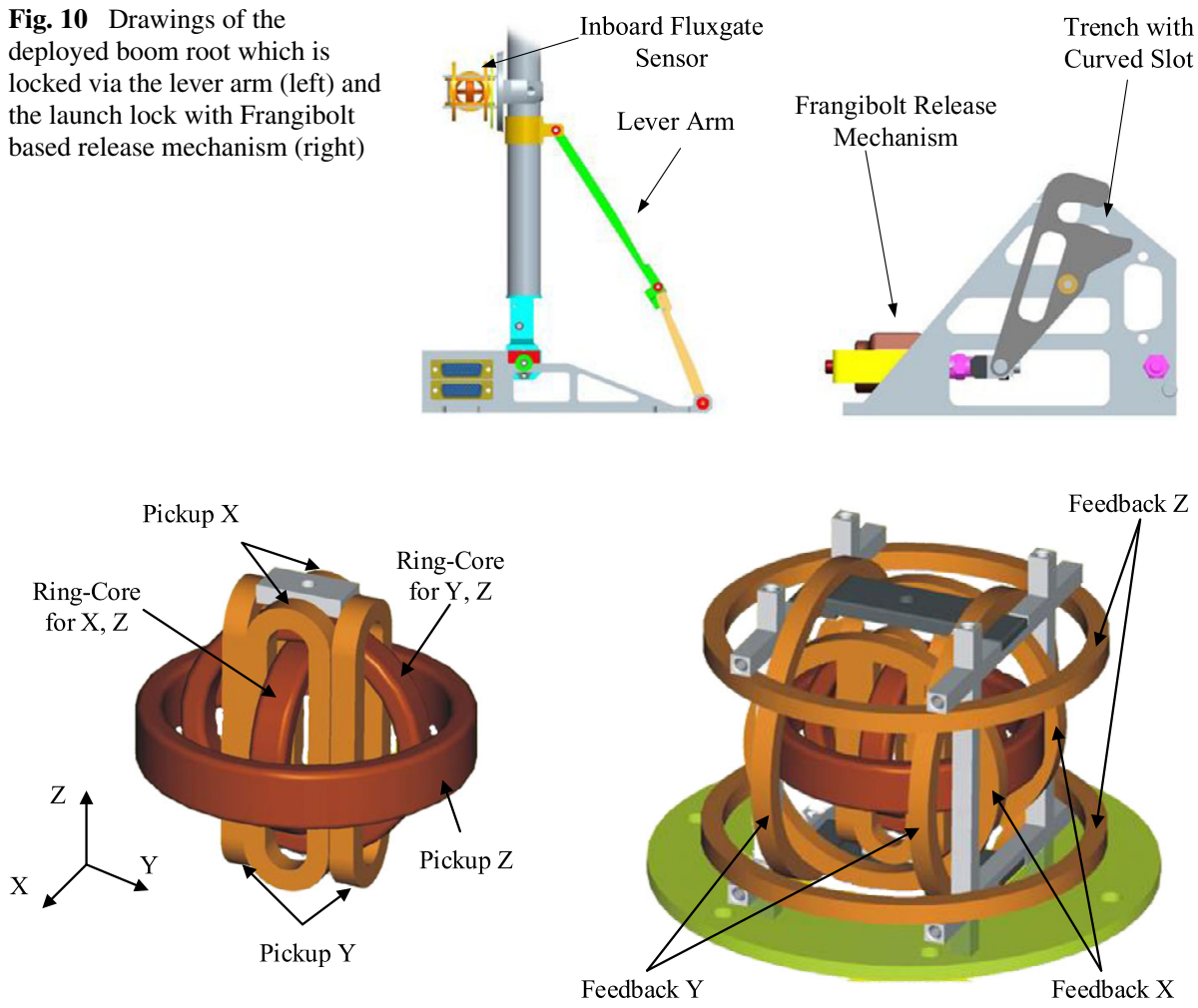

Fig. 11 3-D models of the fluxgate sensor with ring-cores and pickup coil system on the left and a fully functional sensor including the triaxial Helmholtz feedback coils on the right (from Auster et al. 2008)

Iron, $81 \%$ Nickel and 6\% Molybdenum, which has a thickness of $20 \mu \mathrm{m}$. They have a different diameter so that the smaller core can be placed perpendicularly within the larger one to reduce size and mass of the sensor unit. The excitation coils are wound directly on the ring-cores to produce high excitation fields for a proper saturation of the soft-magnetic material. One pair of copper wire based pickup coils is placed around the larger core for sensing the magnetic field along the Y direction as shown in the left drawing of Fig. 11. Another pair of pickup coils does the sensing along the $X$ direction around the smaller core and one pickup coil for the $\mathrm{Z}$ direction envelopes both ring-cores. A 3-axes Helmholtz coil system 
is put over the inner part of the sensor. It generates the feedback field for the compensation of the environmental magnetic field at the centre of the sensor which increases the linearity and stabilizes the alignment. The feedback system is made from Copper and Aluminium only. Both materials have thermal expansion coefficients of about 20 ppm per Kelvin which guarantees a wide temperature range and a temperature independent axes alignment. The ring-cores have been used under extreme environmental conditions aboard numerous space missions as well as in applied geophysics.

\section{On-ground Calibrations}

The on-ground calibration of vector magnetometers based on the fluxgate and especially of the AMR principle is mandatory to resolve the relation between the measured and the true magnetic field vector at the position of the sensor. The mathematical relation between the two vectors in the most compact form is expressed by a $3 \times 3$ transformation matrix and an offset vector (e.g., Balogh 2010). The transformation matrix includes information about scale factors, orthogonality and alignment. The latter is the rotation between the orthogonalised magnetic coordinate system of the sensor and e.g. the mechanical coordinate system of the interface plate to which the sensor is mounted to. The orthogonality can vary with sensor temperature. Scale factors and offsets can change with sensor and electronics temperature. The noise floor and the transfer function with amplitude and phase characteristics are calibration parameters which are not directly covered by the mathematical relation discussed above.

The ground calibration of the SOSMAG sensors was done at Magson GmbH with several multi-layer shielding cans (right picture of Fig. 12) and a thermal control chamber, at the Space Research Institute (IWF) with a sensor temperature test facility (left picture of Fig. 12) and at the calibration site of TU-Braunschweig (often referenced as "Magnetsrode") which is built around a $2.5 \mathrm{~m}$ triaxial Braunbek coil facility (Glassmeier et al. 2007 and Fig. 13).

Temperature dependence of the scale factors, the offsets (Fig. 14) and the orthogonality were determined by repeating the calibration steps over a series of uniformly spaced temperature steps from the minimum to the maximum calibration temperature. The sensor and electronics temperature calibrations were done roughly from $-40{ }^{\circ} \mathrm{C}$ to $60{ }^{\circ} \mathrm{C}$ (5 levels) and from $-30{ }^{\circ} \mathrm{C}$ to $60{ }^{\circ} \mathrm{C}$ (6 levels), respectively. High field (within $\pm 60,000 \mathrm{nT}$ with $10,000 \mathrm{nT}$ steps) and low field sequences (within $\pm 9,000 \mathrm{nT}$ with 1,500 nT steps) were used for the calibration with the coil system in Magnetsrode. Even though the scientifically required dynamic range is below $\pm 500 \mathrm{nT}$, SOSMAG has been built for Earth's field range to cover ground testing and unexpectedly large disturbers on the spacecraft especially at the position of the AMR sensors.

A concise summary of the on-ground calibration parameters is listed in Table 5. The SOSMAG instrument is complaint with all performance requirements as outlined in Table 2 except for the noise floor of the AMR sensors which is $<220 \mathrm{pT} \mathrm{Hz}^{-0.5}$ instead of $<150 \mathrm{pT} \mathrm{Hz}^{-0.5}$.

\section{Flight Operation}

After launch of GK-2A from Kourou in French Guiana on 4 December 2018, SOSMAG was first taken into operation with stowed boom on 4 January 2019. Boom release was performed successfully on the same day. SOSMAG was then kept in commissioning mode with all 

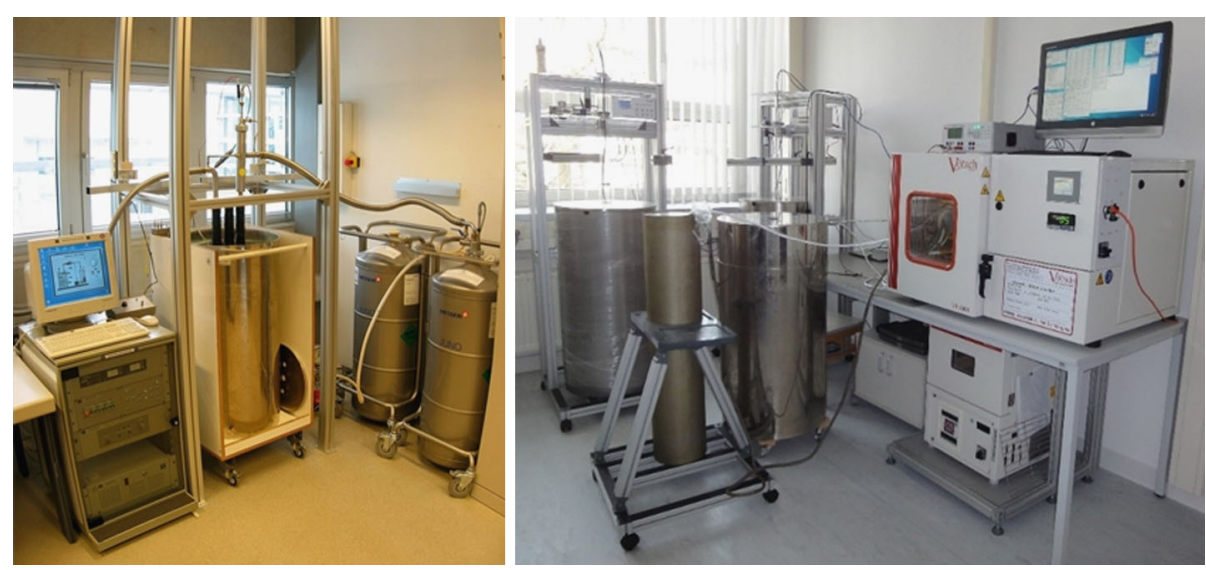

Fig. 12 Pictures of the calibration set-up at IWF (left) and at Magson GmbH (right); The IWF facility enables calibration of offsets, noise, linearity and transfer function for sensors over a temperature range between $-150{ }^{\circ} \mathrm{C}$ and $200{ }^{\circ} \mathrm{C}$ in a low-field environment. The facility consists of a temperature controlled Dewar vessel within a three-layer magnetic shielding and a stimulus coil. At Magson, the magnetic field sensors and the electronics box were placed in three different shielding cans and a temperature control chamber, respectively, for drift measurements at different electronics temperatures
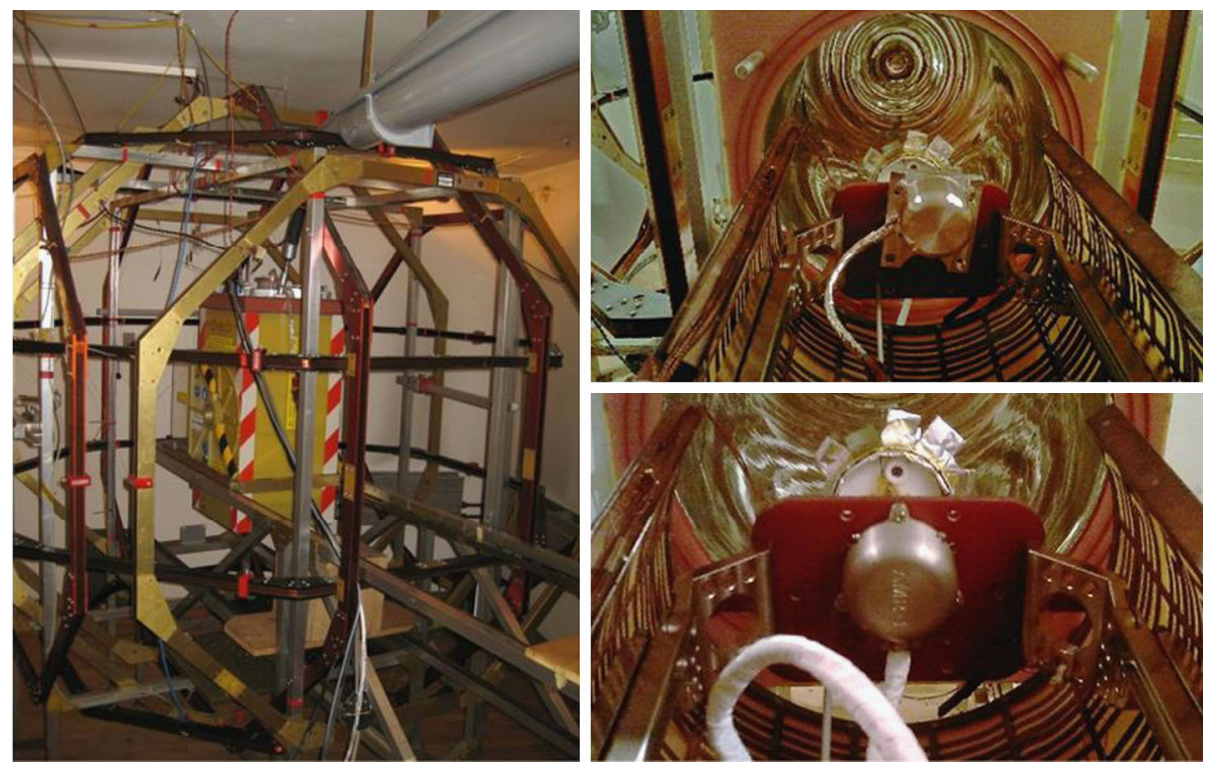

Fig. 13 Braunbek coil system at Magnetsrode with thermal control box in the centre (left) and fluxgate (upper right; before boom installation) as well as AMR sensor (lower right) mounted in the thermal control box

four sensors taking data with 16 vectors per second to monitor the back ground field of the spacecraft up to $8 \mathrm{~Hz}$. On 7 January 2019, the vector rate was reduced to 1 vector per second for all four sensors (instrument mode Normal $1 \mathrm{~V}$ according to Table 4) to open up enough telemetry bandwidth for the commissioning of the KSEM particle sensors. From 25 January 

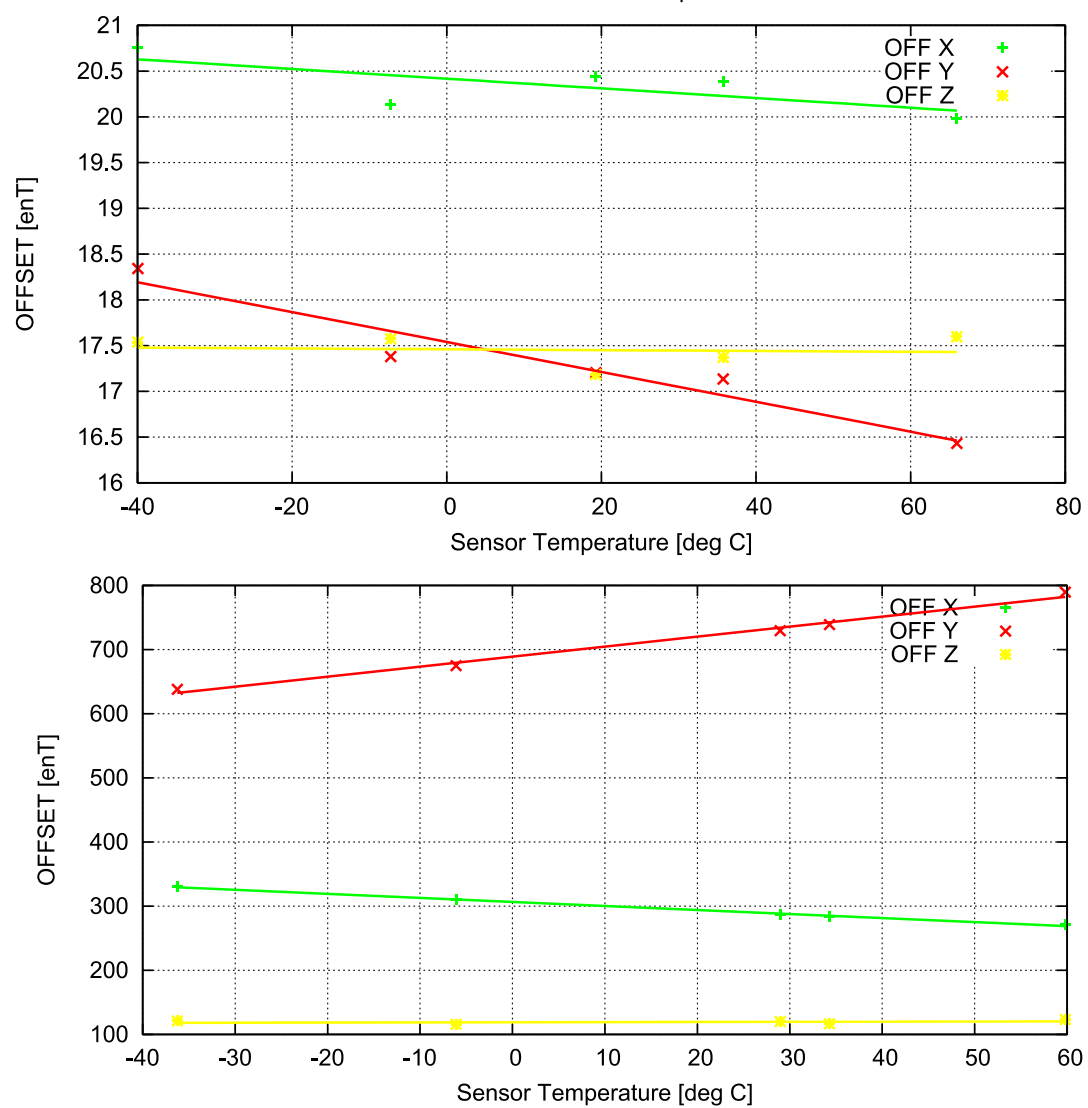

Fig. 14 Drift of the sensor offsets with changing sensor temperature; the maximum drift of the outboard fluxgate (upper plot) and the AMR 1 (lower plot) sensors over a temperature range of about $100{ }^{\circ} \mathrm{C}$ is less than $2 \mathrm{nT}$ and $156 \mathrm{nT}$, respectively

until 25 February 2019 all KSEM sensors were powered down for the commissioning of the Advanced Meteorological Imager, the primary payload of GK-2A.

From 25 February 2019, SOSMAG has been operating in Normal $1 \mathrm{~V}$ almost continuously. The first parameter upload for the on-board data cleaning took place on 2 May 2019.

The following subsections describe the required coordinate systems, the removal of the magnetic disturbances from the spacecraft with the four-sensor approach, the determination of the low frequency offsets by a comparison with the Tsyganenko Earth's field model 2004, two examples of scientific events measured by SOSMAG and the data product flow.

\subsection{Coordinate Systems}

The magnetic field at each sensor is measured in its specific single sensor reference frame and transformed to the reference frame of the outboard fluxgate sensor (FG OB). The four vectors in this common outboard sensor reference frame (OSRF) are then used for the cleaning of the dynamic (AC) disturbances, yielding the cleaned vector in OSRF (B_ACclean). The final vector for physical interpretation shall be given in the physical reference frame 
Table 5 Calibration parameters which were determined on ground

\begin{tabular}{lll}
\hline Parameter & Performance & \\
\cline { 2 - 3 } & FG 1,2 & AMR 1,2 \\
\hline Abs. value of offsets at room temperature & $<21.4 \mathrm{nT} ;$ & $<730.5 \mathrm{nT}$ \\
Noise density at room temperature and 1 Hz & $<10 \mathrm{pT} \mathrm{Hz}^{-0.5} ;$ & $<220 \mathrm{pT} \mathrm{Hz}^{-0.5}$ \\
Offset drift with electronics temperature & $<0.043 \mathrm{nT}^{\circ} \mathrm{C}^{-1} ;$ & $<0.19 \mathrm{nT}^{\circ} \mathrm{C}^{-1}$ \\
Offset drift with sensor temperature & $<0.016 \mathrm{nT}^{\circ} \mathrm{C}^{-1} ;$ & $<1.56 \mathrm{nT}^{\circ} \mathrm{C}^{-1}$ \\
Gain drift with electronics temperature & $<26 \mathrm{ppm}^{\circ} \mathrm{C}^{-1} ;$ & $<13 \mathrm{ppm}^{\circ} \mathrm{C}^{-1}$ \\
Gain drift with sensor temperature & $<20 \mathrm{ppm}^{\circ} \mathrm{C}^{-1} ;$ & $<4.9 \mathrm{ppm}^{\circ} \mathrm{C}^{-1}$ \\
Orthogonality drift with sensor temperature & $<0.19^{\prime \prime}{ }^{\circ} \mathrm{C}^{-1} ;$ & $<3.1^{\prime \prime}{ }^{\circ} \mathrm{C}^{-1}$ \\
3 dB corner frequency of $128 \mathrm{~Hz}$ data & $57 \mathrm{~Hz} ;$ & $28 \mathrm{~Hz}^{-1}$ \\
3 dB corner frequency of $4 / 1 \mathrm{~Hz}$ data & $1.76 / 0.44 \mathrm{~Hz} ;$ & $1.76 / 0.44 \mathrm{~Hz}^{-1}$ \\
Linearity $^{\mathrm{b}}$ & $<4 \times 10^{-5}$ & $<6 \times 10^{-5}$ \\
\hline
\end{tabular}

${ }^{a}$ Maximum linear regression coefficient of all six components of one type of sensor

${ }^{\mathrm{b}}$ For a dynamic range of $\pm 10,000 \mathrm{nT}$

Hpen, with axes directions only depending on the geographical position of the spacecraft, and in the dynamic Geocentric Solar Ecliptic reference frame (GSE), with axes defined by the direction to the Sun, i.e. changing with position of the Earth relative to the Sun.

The physical reference frame Hpen is defined as follows: Hp is the magnetic field component tangent to the Earth meridian, positive to the North Pole; the component $\mathrm{He}$ is pointing to the Earth, positive to Nadir, and $\mathrm{Hn}$ is normal to the above, tangent to the parallel circle and positive to East.

The standard transformation from OSRF to the Hpen reference frame for the nominal attitude of GK-2A ( $\mathrm{X}_{\mathrm{SC}}$ in direction of the spacecraft's velocity positive to East, $\mathrm{Y}_{\mathrm{SC}}$ tangent to meridian positive to South Pole and $\mathrm{Z}_{\mathrm{SC}}$ positive to Nadir) is given by the equations:

$$
\begin{array}{ll}
\mathrm{Hp}=-\mathrm{By}_{\mathrm{OSRF}} & \mathrm{Hp}=-\mathrm{By}_{\mathrm{SC}} \\
\mathrm{He}=\mathrm{Bx}_{\mathrm{OSRF}} & \mathrm{He}=\mathrm{Bz}_{\mathrm{SC}} \\
\mathrm{Hn}=-\mathrm{Bz}_{\mathrm{OSRF}} & \mathrm{Hn}=\mathrm{Bx}_{\mathrm{SC}}
\end{array}
$$

The dynamic GSE reference frame has its $X_{\mathrm{GSE}}$ axis pointing from Earth to Sun, $\mathrm{Y}_{\mathrm{GSE}}$ axis opposite to the orbital velocity of Earth and $\mathrm{Z}_{\mathrm{GSE}}$ axis normal to $\mathrm{X}_{\mathrm{GSE}}$ and $\mathrm{Y}_{\mathrm{GSE}}$. The transformation from OSRF to GSE requires the longitude of the Earth relative to the equinox and the obliquity of the ecliptic, both in the standard reference frame and epoch J2000.

\subsection{Removal of Dynamic Magnetic Disturbances}

The short length of the boom and the absence of magnetic cleanliness measures result in rather strong spacecraft-generated dynamic (AC) magnetic disturbances at all sensors. However, multi-sensor measurements allow for the removal of these disturbances. While the ambient magnetic field is the same at all sensors, independent on their position, the measured magnitude and direction of each artificial disturbance depends on the position of the sensor where it is measured. As a result, the difference $\Delta \boldsymbol{B}^{0, i j}=\boldsymbol{B}^{0, i}-\boldsymbol{B}^{0, j}$ between measurements taken by sensors $i$ and $j$ contains the spacecraft disturbances without any contribution 
from the ambient magnetic field. It can be shown that pure single dipole or quadrupole contributions from on-board magnetic field disturbers can always be corrected for by applying an appropriate linear transformation to the $\Delta \boldsymbol{B}^{0, i j}$ differences:

$$
\boldsymbol{B}_{\text {corrected }}^{i}(t)=\boldsymbol{B}^{0, i}(t)+\mathbf{A}^{i j} \Delta \boldsymbol{B}^{0, i j}(t)
$$

where the coefficient matrix $\mathbf{A}^{i j}$ depends only on the positions of the magnetic field source and of the sensors.

In contrast to the single multipole disturber case, there is no general recipe to remove more complex disturbances, i.e. disturbances originating from multiple sources with random multipole contributions placed at different positions. Fortunately, most artificial magnetic field disturbances on-board spacecraft are far from random. Some of the characteristics of the artificial disturbers can help isolate their contribution to the measured magnetic field. One method taking advantage of commonly encountered properties of magnetic disturbers on-board spacecraft is the Principal Component Gradiometer (PiCoG) method described by Constantinescu et al. (2020) which we use to clean the magnetic field data acquired by SOSMAG.

The PiCoG method can remove up to three linearly polarized disturbances for each pair of sensors and it is based on the assumption that each disturber can be represented by a time varying magnetic dipole or quadrupole which keeps constant orientation. At the sensor locations, such disturbers will produce linearly polarized magnetic fields and therefore each disturbance will only affect one component of the measured magnetic field if represented in a coordinate system aligned with its local polarization direction. Multiple disturbances coming from locations far apart from each other can be removed only if their polarization directions are mutually orthogonal. Non-orthogonal disturbances can be nevertheless removed together if they come from sources close to each other compared with the distance to the sensors. To identify the polarization directions of the disturbances it is further assumed that, at time scales characteristic to each disturbance, the maximum variance direction of the measured magnetic field is determined by the targeted disturbance.

If the conditions stated above are met, then the coefficient matrix $\mathbf{A}^{i j}$ can be estimated based only on the measurements taken by the sensors $i$ and $j$. For each pair of sensors the corrections are applied iteratively. The $n$-order correction of the measurements taken by sensor $i$ using measurements taken by sensor $j$ can be written as:

$$
\boldsymbol{B}^{n, i j}=\boldsymbol{B}^{n-1, i j}+\mathbf{A}^{n-1, i j} \Delta \boldsymbol{B}^{n-1, i j} ; \quad n=1, \ldots, 3 ; \boldsymbol{B}^{0, i j}=\boldsymbol{B}^{0, i}
$$

The corrected values replace then the measurements of the next sensor pair until all disturbances are removed.

One must be aware that the correction does not come without cost. Disturbances present at only one of the sensors, such as sensor specific noise or offset oscillations triggered by environment changes, are transferred to the corrected measurements either reduced or enhanced, depending on the geometry of the sensors-source system. As a rule of thumb, the amount of the introduced perturbations is roughly proportional to the ratio between the magnitudes of the variations of the disturbance being corrected at the two sensors. Similarly, perturbations with a spatial variation different from the spatial variation of the targeted disturbance will be transferred to the corrected measurements. This can be e.g. a quadrupole disturbance superposed on a dipole disturbance to be removed. Therefore it is best to remove the disturbances using the minimum necessary number of steps.

The magnetic field measurements taken by the FG sensors on 04 March 2019 between 13:00 and 21:00 UT and the correction result are displayed in Fig. 15. The disturbances 

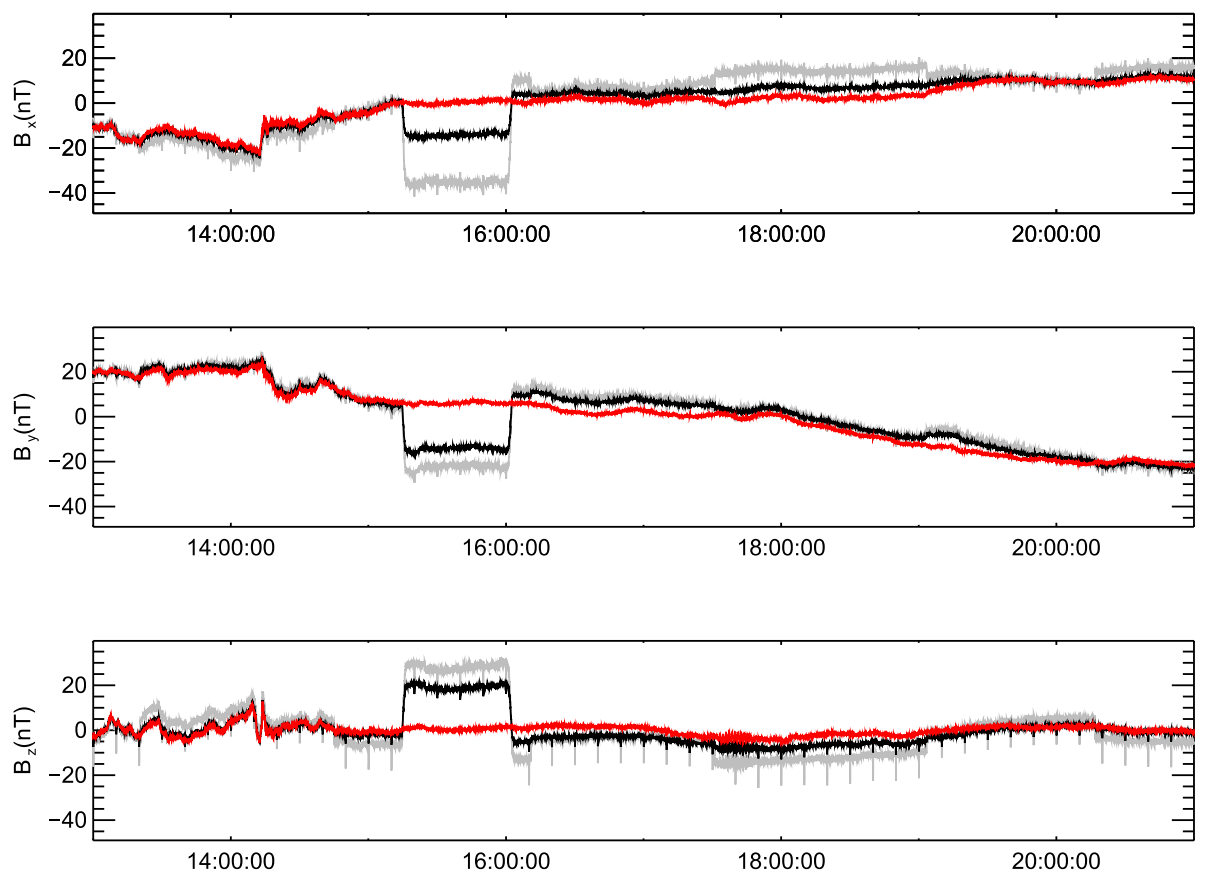

Fig. 15 The magnetic field in OSRF measured on 04 March 2019 by the FG IB (grey lines) and FG OB (black lines) together with the final result of the AC correction (red lines)

have larger amplitudes at the inboard sensor but both sensors are affected. Below we discuss four types of disturbances which we removed from the measured data: the large disturbance starting shortly after 15:00 UT, the step-like disturbances such as the ones around 17:30 UT and 19:00 UT, the spikes repeating every $10 \mathrm{~min}$, and the noise which can be seen in the top panel of Fig. 16, affecting a broad frequency band higher than the characteristic frequencies of the other disturbances, corresponding to periods from minutes up to the Nyquist frequency of the $1 \mathrm{~s}$ resolution data.

The largest disturbance begins suddenly around 15:15 UT as a step of several tens of nT in all components. Because 15:00 UT corresponds to the local midnight, we refer to this disturbance as the Midnight Disturbance (MD). The disturbance remains high until around 16:00 UT when it drops back, overshooting by about $5 \mathrm{nT}$, keeping the nearly constant value until $4 \mathrm{~h}$ later at 20:00 UT when it ends. This disturbance appears only once per day and affects much more the AMR sensors then the FG sensors, reaching an amplitude roughly 5 times larger at AMR 2 and 40 times larger at AMR 1.

Because the AMR sensor specific noise - which is 20 times larger than the FG sensor noise - is going to be introduced inversely proportional to these factors, we pick the AMR 1 sensor to correct this disturbance at both FG sensors. All other disturbances detected by the AMR sensors are much smaller and using AMR data to correct them would therefore introduce unacceptable high noise levels. The disturbance is removed in one iteration from FG OB and FG IB and the result is used as initial data for the next step.

The next disturbance to be removed is the higher frequency (periods less than several minutes) disturbance visible in the top panel of Fig. 16 as a constant broadband noise enhanced around $0.3 \mathrm{~Hz}$ and below $0.2 \mathrm{~Hz}$ over the entire data interval of one day. To isolate 

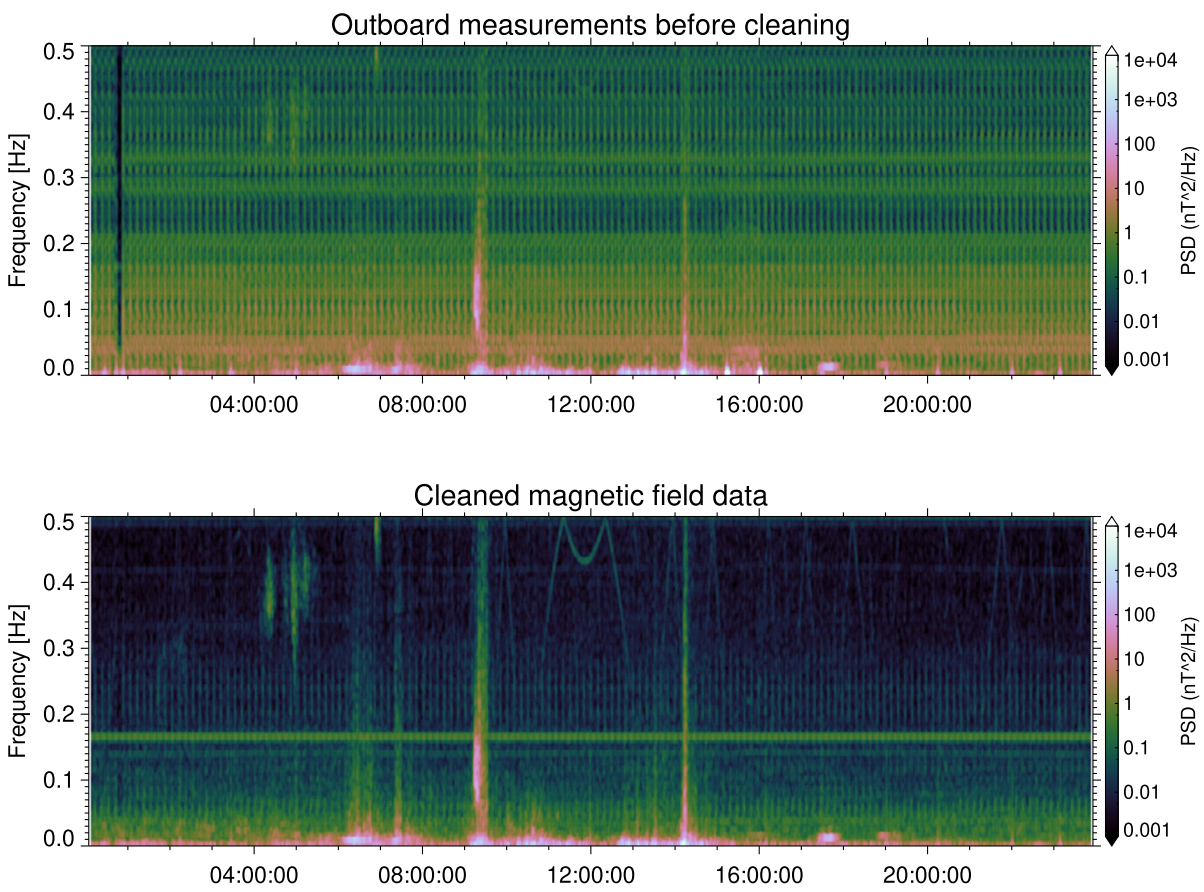

Fig. 16 Total spectral power density of the magnetic field data before and after AC cleaning on 04 March 2019

this disturbance we use a sliding window of $100 \mathrm{~s}$, below the characteristic time scales of all other targeted disturbances. From the resulting ensemble of cleaning parameters we compute the correction matrix $\mathbf{A}^{0, i j}$ which is then used to remove this disturbance by the first order correction.

The resulting first order corrected data is then processed using a window of $700 \mathrm{~s}$ to determine the cleaning parameters for the spikes which are then removed by the second order correction.

The last disturbance to be removed are the steps occurring at non-regular intervals slightly larger than one hour. A window length of $16,000 \mathrm{~s}$ is used to insure that at least one step is always included.

Table 6 lists the magnitudes of the identified disturbances as measured initially by FG $\mathrm{OB}$ and after the full AC correction (single order AMR1-FGOB/AMR1-FGIB plus third order FGOB-FGIB). The remnants of the MD are less than $2 \mathrm{nT}$. These were determined as the difference between the medians over $90 \mathrm{~s}$ intervals immediately before and after the ramps. The remnants from the steps, determined in a similar fashion are about $1.3 \mathrm{nT}$. The mean magnitude of the spikes after cleaning, which is determined as the difference between the spike peak and the median over the $20 \mathrm{~s}$ intervals before and after the spike, is reduced to about $0.3 \mathrm{nT}$. The remaining magnitude of the high frequency disturbance is determined as the peak-to-peak amplitude of the disturbed data from which the peak-to-peak amplitude during a time interval when the disturbance is absent is subtracted.

Not all AC disturbances can be corrected using the PiCoG method. One class of such disturbances is represented by the sensor offset oscillations ( $\mathrm{SOO}$ ) triggered by large temperature gradients. The accommodation of the SOSMAG boom combined with its short 
Table 6 The magnitude of the identified disturbances as initially measured by the FGOB and the final magnitudes after $\mathrm{AC}$ cleaning. The magnitudes of the MD and of the steps are defined as the mean size of the ramps. The magnitude of the spikes is defined as the mean size of the spikes. The magnitudes of the high frequency disturbance, of the $6 \mathrm{~s}$ tone and of the reaction wheels disturbance are computed as peak-to-peak amplitudes. The magnitudes of the MD edges and for the SOO are defined as maximum peak-to-peak values

\begin{tabular}{lll}
\hline Disturbance class & FG OB & Corrected \\
\cline { 2 - 3 } & $(\mathrm{nT})$ & $(\mathrm{nT})$ \\
\hline Corrected using PiCoG & & \\
MD leading ramp & 33.59 & 1.81 \\
MD trailing ramp & 39.97 & 1.15 \\
Steps & 2.54 & 1.30 \\
Spikes & 4.93 & 0.30 \\
High frequency & 0.86 & 0.09 \\
Not corrected & & \\
SOO & 4.00 & 5.00 \\
MD edges & 2.00 & 2.00 \\
6 s tone & 0.05 & 0.08 \\
Reaction wheels & 0.04 & 0.04 \\
\hline
\end{tabular}

length in relation to the size of the GK-2A spacecraft (Fig. 6) has resulted in unexpected shadowing of the entire boom for more than 8 hours every day. The temperature of the outboard fluxgate sensor drops from $+30{ }^{\circ} \mathrm{C}$ to $-90{ }^{\circ} \mathrm{C}$ during this period. Since the sensor was not designed and the ring-cores were not pre-selected for these extreme temperature changes, SOO with peak-to-peak values up to $4 \mathrm{nT}$ in the FG OB measurements before correction are triggered. Because of the factors applied to the FG OB measurements, the PiCoG correction actually increases the SOO disturbance up to $5 \mathrm{nT}$. The oscillations for ten days in a row during Aug. 2019 are illustrated in Fig. 17.

Another disturbance class which could not be treated by PiCoG are disturbances produced by sources which are located too far from the AMR sensors and therefore the signal ratio between the AMR and the FG sensors is smaller than the noise ratio (about 20) between the sensor types. Three of those interferences could be identified: A triangular-shape pulse centred on the MD ramps, lasting about three minutes and reaching an amplitude of about $2 \mathrm{nT}$, a tone around $0.166 \mathrm{~Hz}(6 \mathrm{~s}$ period) with a peak-to-peak amplitude of about $0.08 \mathrm{nT}$ showing up in the spectrogram of the cleaned data, and a disturbance generated by the reaction wheels, with a peak-to-peak amplitude of $0.04 \mathrm{nT}$ visible around noon in the spectrogram of the cleaned magnetic field data in Fig. 16.

An overall measure of the effectiveness of the cleaning is the mean reduction of the Power Spectral Density (PSD) over a given frequency range. For periods in the range of 2 sec to 1 min the mean PSD of the cleaned data is 7.8 times lower than the mean PSD of the initial measurements of the outboard fluxgate sensor. Since the presence of the ambient magnetic field only worsens this ratio, we conclude that in this period range the PiCoG cleaning reduced spectral power of the disturbances at least by a factor of 7.8. For longer periods, in the range of 1 min to 6 hours, we find a reduction factor of the spectral power of at least 3.9.

Because the correction consists simply of a linear combination of the measured values, it can be applied on-board, allowing for real-time delivery of cleaned magnetic field data. To limit updating of the on-board software, of key importance is the stability of the cleaning parameters. The stability was examined by computing the standard deviation of the cleaning parameters determined for every Sunday with available data in 2019. The maximum change in the corrected magnetic field corresponding to the computed deviations was less than $0.2 \mathrm{nT}$. 


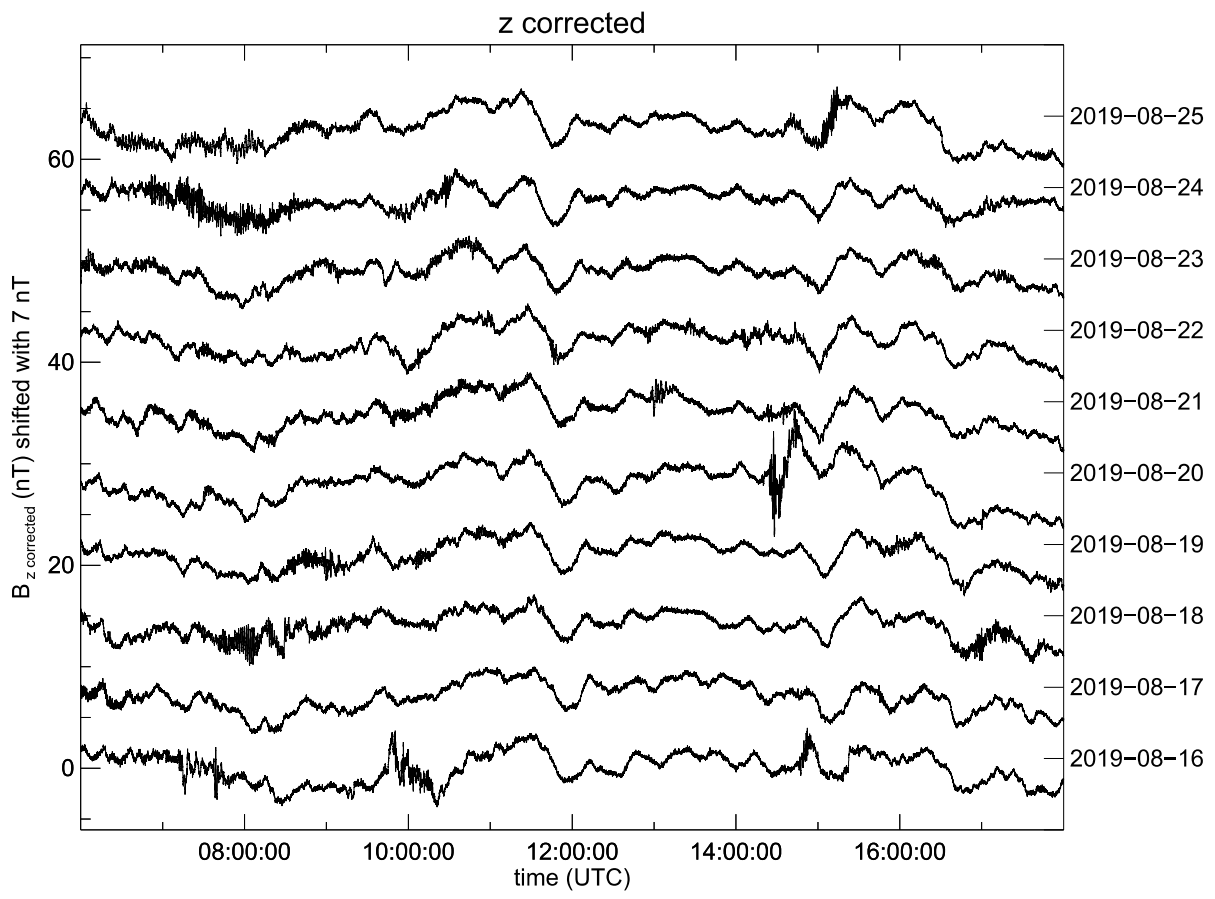

Fig. 17 Example of sensor offset oscillations triggered by temperature gradients around dawn during ten consecutive days with relatively quiet geomagnetic conditions

\subsection{Offset Determination and Alignment}

After correction for the dynamic (AC) spacecraft disturbances, an unknown static offset contribution is still contained in the data: the $\mathrm{AC}$ cleaned data are deviating from the real space field only by a constant or slowly varying offset in all three components. Each "DC offset" is a sum of three contributions: the offset of the sensor, the spacecraft background DC field and an offset induced through the AC correction, since fractions of the main DC level at the other sensors are subtracted from the outboard fluxgate sensor during the cleaning process. It is impossible but also not necessary to determine each single contribution separately; only the total sum of the contributions needs to be processed for each component.

The DC offsets are determined through comparison of the AC cleaned data, averaged to 1 min. resolution, with model data for times with low geomagnetic activity by a selection of data with low Kp-index values (taken from ftp.swpc.noaa.gov/pub/indices). The Tsyganenko model 2004 (Tsyganenko 1989; Tsyganenko and Sitnov 2005) is chosen as a standard. It allows the use of measured solar wind parameters from e.g. the WIND spacecraft (Lepping et al. 1995) for the calculation of the magnetic field at large distances from the Earth, i.e. here at the geostationary position of the GK-2A spacecraft and for the time when the magnetic field was measured by SOSMAG. The Tsyganenko model (T04) uses the Hpen reference frame. To enable a proper comparison, all AC cleaned data are transformed to Hpen too.

As an example, Fig. 18 shows the AC cleaned SOSMAG data compared to T04 for November 2019. The observed and model data have been selected for times with Kp-index $\leq 1.0$ and the differences between them were calculated. The medians of the differences 


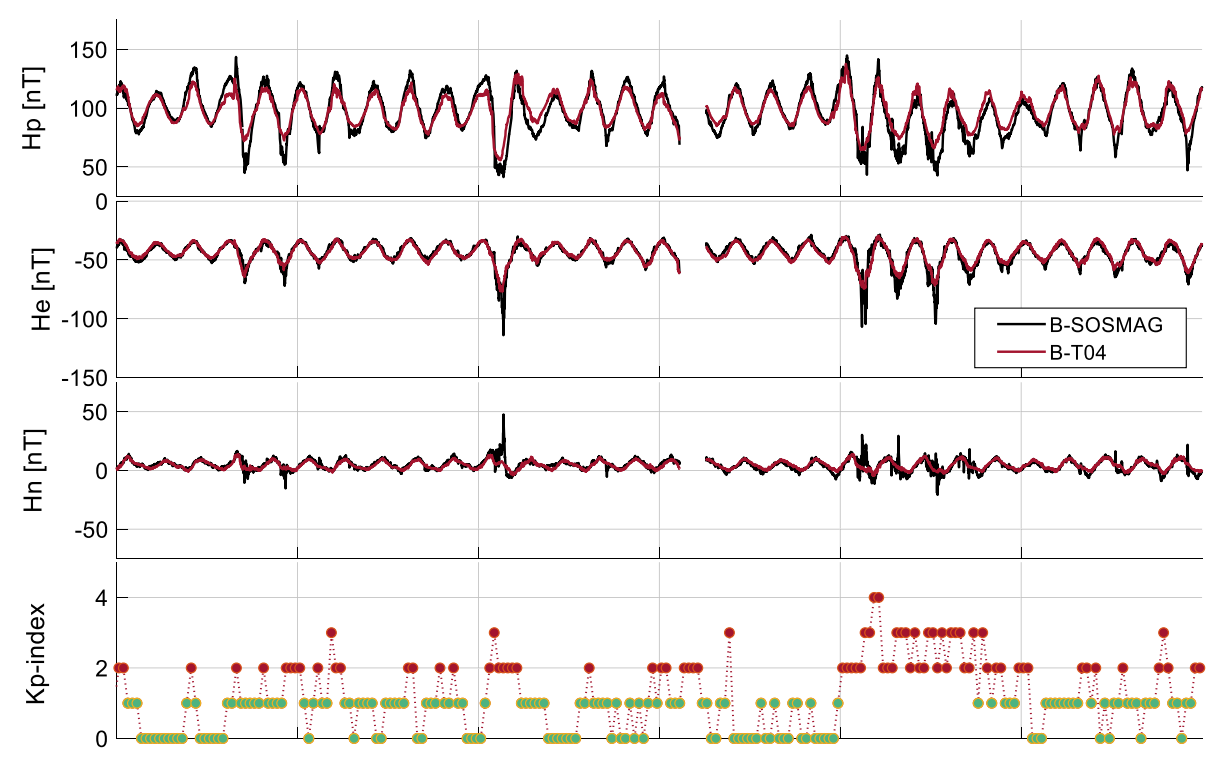

2019-11-01 00:00 to 2019-11-30 23:59 [UTC]

Fig. 18 AC cleaned and DC offset corrected data for November 2019 in Hpen (upper three panels, black lines) compared to the Tsyganenko model data (red lines). DC offsets $(14.8,38.4,-89.8 \mathrm{nT})$ were determined from selected times with $\mathrm{Kp} \leq 1.0$ (shown in bottom panel, $68.0 \%$ of data selected) as median of the difference between observed and model magnetic field data. The standard deviation of the difference between the two plotted data sets for $\mathrm{Kp} \leq 1.0$ is 6.7, 2.3 and $2.0 \mathrm{nT}$ for $\mathrm{Hp}$, He and $\mathrm{Hn}$, respectively

were adopted as DC offsets valid for this time interval. The offsets were subtracted from the data to yield the correct data level.

Since the values of the DC offsets were determined only from the restricted data set with $\mathrm{Kp}$-index $\leq 1.0$, it is clear that the data obtained at times with active magnetosphere may deviate substantially from the model. Especially the Hp component shows systematic deviations from the model data. For example, the minimum and maximum values of the Hp component are lower and higher, respectively, in the observed data than in the model data.

Moreover, looking closer at the difference between the black and red lines for the Hp component in Fig. 18, there seems to be a systematical difference depending on the day time also during days with quiet magnetosphere. This could be due to day time dependent disturbers on the spacecraft. To check this in more detail, the differences between the AC cleaned and the T04 model data were investigated for 10 months (2019-03 to 2019-12); per month, the mean values of the difference were determined as a function of local day time (geographical longitude of GK-2A spacecraft is $128.2^{\circ}$ East). The results are shown in Fig. 19, left three panels, which clearly reveal a systematic variation as a function of local time. Especially the deviation of the Hp component from the model is varying with local time by 8 to $10 \mathrm{nT}$ peak-to-peak. The variation has a minimum for local night times and a broadened maximum around local noon. Overall, the He component shows an inverse trend with the maximum of the variation, which is about $5 \mathrm{nT}$ peak-to-peak, around local mid night and the minimum before noon. The daily variation is clearly the smallest in Hn with just 1-2 nT peak-to-peak and no clear trend during the day.

Since from this result it cannot be distinguished if the daily variation is generated by the sensors, the spacecraft or the model, we did the same analysis with the data from the 

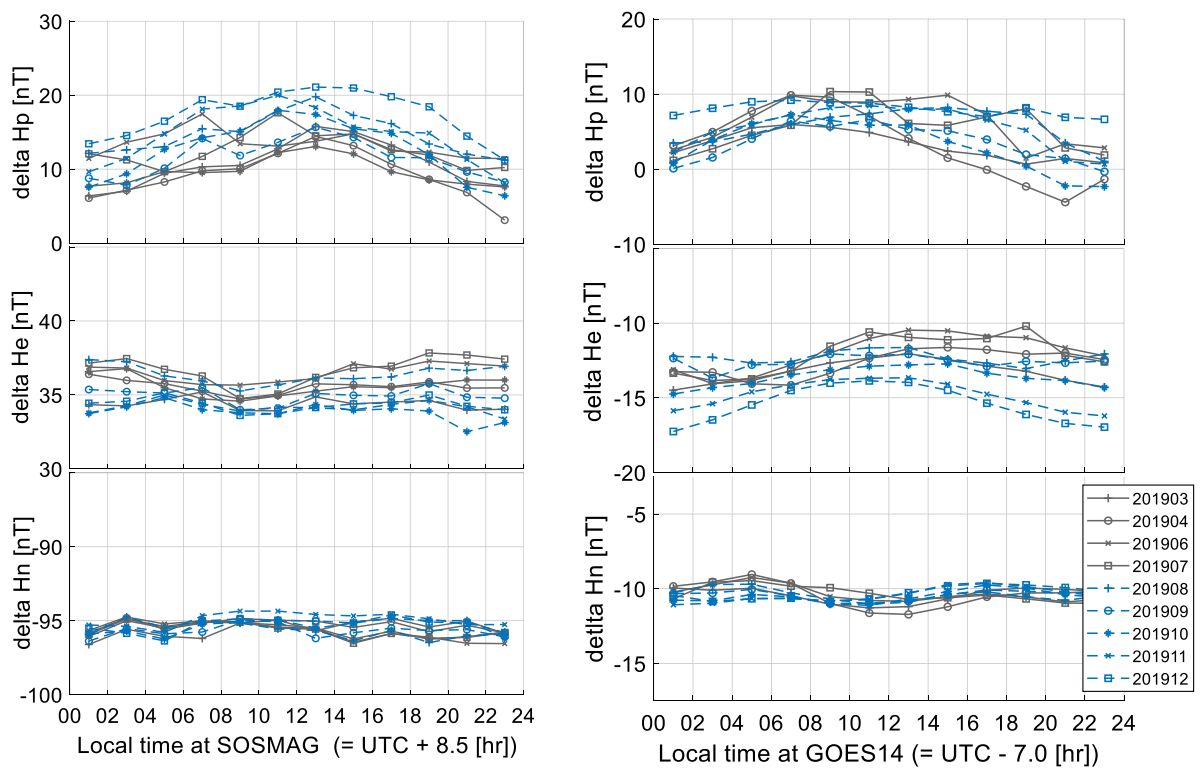

Fig. 19 Left: SOSMAG AC cleaned data difference to T04 as a function of local time at the spacecraft for 2019-03 to 2019-12 with one curve per month. Right: GOES-14 data difference to T04 as function of local time, for 2019-03 to 2019-12 with one curve per month. The scale of the $y$-axis is the same in all six panels

GOES-14 magnetometer. The GOES-14 satellite is at geostationary position with $255.6^{\circ}$ eastern longitude (Singer et al. 1996; GOES-14 data from www.swpc.noaa.gov/products/ goes-magnetometer). Again, the model magnetic field is calculated with T04 using the WIND data as input for the solar wind conditions. The three panels on the right of Fig. 19 show the deviation of the GOES-14 data from the model as a function of local time at the spacecraft. A dependence of the differences on the local time can be seen here as well.

Similar to SOSMAG, the daily variation in the Hp component is about $8 \mathrm{nT}$ peak-topeak with the minimum at local midnight and a broadened maximum around noon. The He component also shows a significant daily variation (5-8 nT peak-to-peak) but with the maximum around local noon, as for Hp. The variation in $\mathrm{Hn}$ is less than $5 \mathrm{nT}$ peak-to-peak but with two maxima during 24 hours. It is also obvious that the GOES-14 data do not vary around zero nanotesla which indicates that the GOES-14 offset processing is not linked to the T04 model.

The daily variations of GOES-14 compared to SOSMAG are about the same in Hp but different in $\mathrm{He}$ and $\mathrm{Hn}$. This may be explained by the tilt of the magnetic axis relative to the rotational axis of the Earth with GOES-14 being located slightly north of the magnetic equator and GK-2A slightly south of it.

Taking into account that the peak-to-peak variation is nearly the same for both, measurements on GOES-14 and GK-2A, it can be concluded that the local time dependence of the deviation of the SOSMAG data from the model data is to a large extend neither caused by the SOSMAG sensors nor by spacecraft interferences but is inherent to the model data. In other words, the daily variation of the Tsyganenko model data is not fully representing the real daily variation at the position of the spacecraft. A similar effect was reported for the GOES-16 magnetometer data (Loto'aniu et al. 2019). A comparison of these data with the 


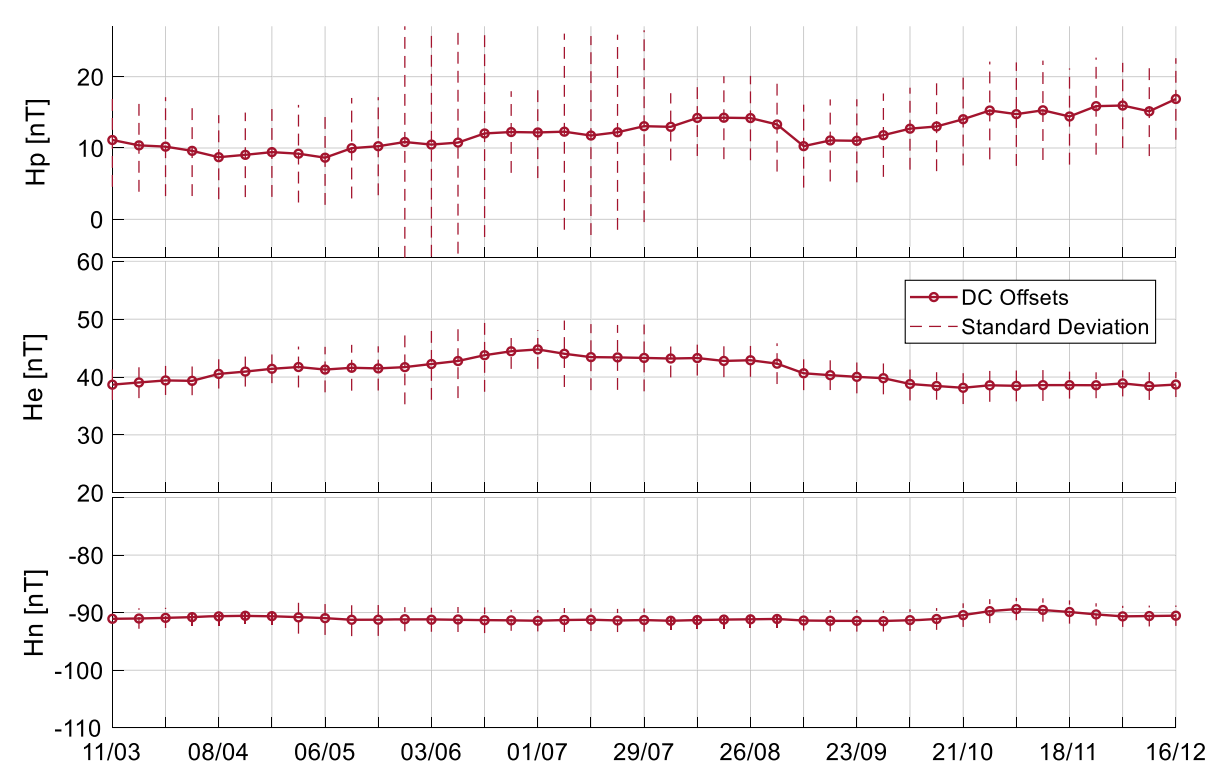

Fig. 20 Final values for DC offsets (in Hpen) determined from times with Kp-index $\leq 1.0$ for 10 months of data (2019-03 to 2019-12) using sliding windows; same scale on $y$-axis in all panels. Standard deviations were determined from the selected data points $(\mathrm{Kp} \leq 1.0)$ only

T04 model revealed differences to the model, where the Hp component showed the strongest and diurnally varying deviations.

To enable a smooth transition of the final SOSMAG offsets from month to month, it was decided to use a sliding window approach for the processing. A rectangular window with a length of 28 days was chosen for each DC offset determination. The window is shifted by seven days for the next calculation, leading to a value every seventh day. For all data in 2019 the thus obtained DC offsets are shown in Fig. 20. The offsets vary with less than about $8 \mathrm{nT}$ in Hp, $5 \mathrm{nT}$ in He and $2 \mathrm{nT}$ in Hn. The behaviour among the three components is comparable to the daily variations in Fig. 19 for which the largest and the smallest variation are also seen in $\mathrm{Hp}$ and $\mathrm{Hn}$, respectively. In general, the long-term variation is fairly low and as such not much worse compared to offset drifts of e.g. a magnetospheric mission like Cluster (Alconcel et al. 2014). The subtraction of the DC offsets from the AC cleaned data is done with linear interpolation between the calculated offset points to produce the fully corrected SOSMAG data.

The final SOSMAG data were compared with the magnetic field measurements aboard the four-satellite Magnetospheric Multiscale (MMS) mission (Burch et al. 2016) on a specific day when the highly elliptical orbits of the MMS satellites came close to the position of GK-2A. The main goal of the MMS mission is to investigate the small-scale physics of magnetic reconnection. Therefore, the four spacecraft fly in tight tetrahedral configuration along a common orbit. To achieve their goal, highly accurate magnetic field measurements with accuracies better than $0.5 \mathrm{nT}$ are required (Russell et al. 2016; Torbert et al. 2016a). Each MMS spacecraft is equipped with two science grade magnetometers mounted on the tips of two five meter long booms. The spin stabilization of the MMS spacecraft enormously aids the in-flight calibration of the magnetometers (Plaschke et al. 2019), including the determination of the spin plane offsets to accuracies of the order or better than $0.1 \mathrm{nT}$. The 


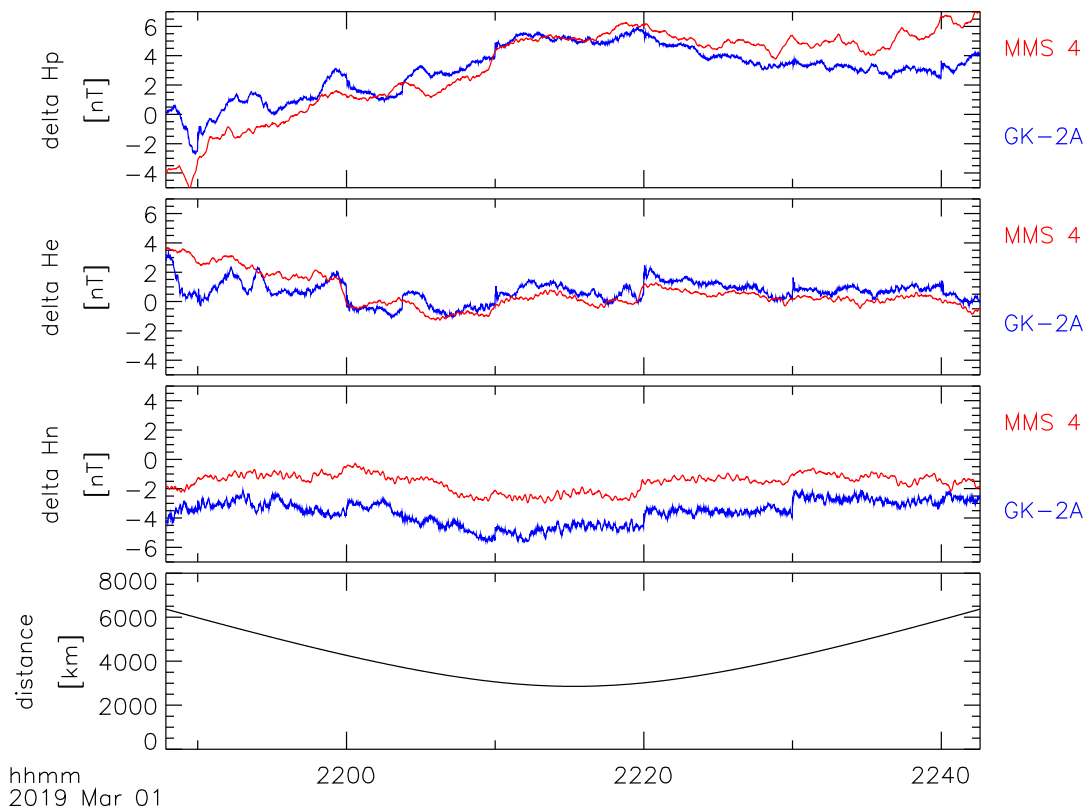

Fig. 21 The purpose of the plot is to assess the uncertainty of the GK-2A offset calibration, by comparing Tsyganenko 2004 model data with GK-2A and Magnetospheric Multiscale (MMS) 4 data when that latter spacecraft was relatively close to GK-2A on 1 March 2019 around 22:15:19 UT. At this time, the distance between the two spacecraft became as low as $2857 \mathrm{~km}$

comparison of the fluxgate magnetometer with electron drift instrument (EDI) data (Torbert et al. 2016b) allows for regular in-flight calibration of the spin axis offsets, as well. Thereby, overall uncertainties on the order of 0.1 to maximally $0.3 \mathrm{nT}$ are regularly achieved in low fields within the regions of interest for the MMS mission (Plaschke 2019). Hence, we can use the MMS data as a standard against which the accuracy of the Tsyganenko model as well as of the GK-2A magnetometer offset calibration can be judged.

For this comparison, we used SOSMAG AC cleaned data of 1 March 2019 of which the offset vector $(10.9,37.6,-90.6) \mathrm{nT}$ in Hpen and the T04 model field were subtracted. The $\mathrm{Kp}$ index at this time was 4 . The result is shown in blue in the top three panels of Fig. 21. In red we show the difference between the fluxgate magnetometer data of MMS spacecraft four and the corresponding T04 field data, also in Hpen coordinates. Within the interval shown, the two spacecraft were located at distances below one Earth radii.

As it can be seen, the instantaneous difference between the measured data and the model field can easily become as large as a few nT. Even the MMS 4 data show this, which we regard as a highly accurate standard for comparison. If the GK-2A magnetometer data were equally accurately calibrated, then we would expect the differences to the model field to be equal to those determined from MMS 4 data during closest approach. For the $\mathrm{Hp}$ and $\mathrm{He}$ components this is indeed approximately the case within MMS accuracy margins. For the Hn component, however, we see a systematic difference between GK-2A and MMS data of approximately $2 \mathrm{nT}$. Hence, at this particular moment, an Hn-component offset value of -92.6 nT would be more accurate.

Taking into account that (a) the daily variations of the SOSMAG offsets relative to the Tsyganenko model are mainly caused by the model, (b) the comparison with the MMS data 


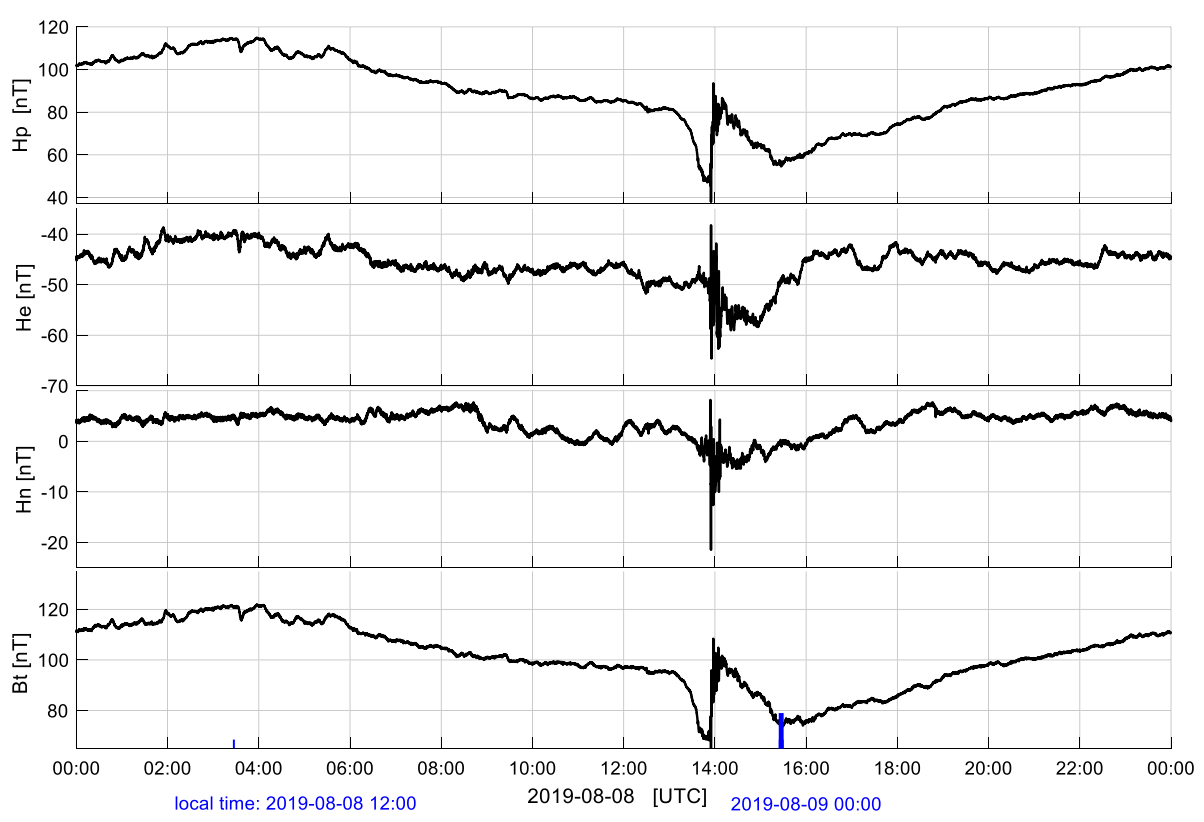

Fig. 22 Effect of a substorm in the magnetotail, observed in the SOSMAG data on 8 August, 2019 around 22:30 local time

on 1 March show a maximal difference of $2 \mathrm{nT}$ and (c) the long-term trend of the offset drift is fairly smooth based on good statistics, we conclude that the low frequency error (signal frequency $<0.1 \mathrm{mHz}$ ) of the SOSMAG final data is below the required $5 \mathrm{nT}$ (cf. Table 1).

The alignment of the cleaned and DC offset corrected data against the Hpen coordinate system of T04 was evaluated for the entire data from 2019. The derived angles (standard deviation in brackets) for the rotation around $\mathrm{Hp}, \mathrm{He}$ and $\mathrm{Hn}$ are $1.7^{\circ}\left(4.0^{\circ}\right),-0.5^{\circ}\left(1.4^{\circ}\right)$ and $-0.5^{\circ}\left(1.9^{\circ}\right)$, respectively. Due to the fact that the standard deviation is by a factor of 2 to 4 larger than the misalignment, it was decided to leave it uncorrected. The investigation has also shown that the alignment accuracy is within the requirement, 10 degrees, of Table 1.

\subsection{Science Observations}

Two science events are discussed in this chapter for an exemplary demonstration that the cleaned SOSMAG data, which are measured on an industry standard satellite without much thoughts on magnetic cleanliness, are very useful to diagnose variations of the magnetic field and to investigate space-weather disturbance events such as magnetic storms and magnetospheric substorms at the geostationary orbit.

Figure 22 shows an example of a magnetospheric substorm recorded by SOSMAG at $128.2^{\circ}$ East longitude, $0^{\circ}$ latitude and $R \approx 6.6$ Re on 8 August, 2019. The northward component (Hp component, top panel) is dominant at the spacecraft location (in the pre-midnight sector with a local time of 22:30) and moderately decreases from about $100 \mathrm{nT}$ at 06:00 UT to $80 \mathrm{nT}$ at 13:00 UT. Then, the Hp component suddenly but smoothly falls down to about $50 \mathrm{nT}$ between 13:00 UT and 14:00 UT and recovers quickly to a level of about $80 \mathrm{nT}$ at 14:00 UT, accompanied by turbulent disturbance in the earthward (He) and eastward ( $\mathrm{Hn})$ 
components. The Hp component falls again to about $60 \mathrm{nT}$ until 15:30 UT and gradually recovers to $100 \mathrm{nT}$ by the midnight (9 August, 2020, 00:00 UT).

At the same time, the MMS spacecraft are located between 00:00-01:00 LT in the Earth's magnetotail approximately $X=-25 \mathrm{Re}$. MMS observes a very disturbed magnetotail with many flow bursts created by magnetic reconnection (substorms; see middle panels of Fig. 23).

As GK-2A moves into the pre-midnight sector of the magnetosphere, the magnetic field starts to decrease at $\sim$ 13:00 UT (approximately 22:30 local time), which corresponds to the growth phase. Looking at the solar wind data in Geocentric Solar Magnetospheric (GSM) coordinate system (Fig. 24), it shows that the Bz component of the Interplanetary Magnetic Field (IMF) becomes negative at $\sim 13: 00 \mathrm{UT}$, which means that dayside reconnection is taking place and field lines are transported to the tail. At the same time ground based magnetometers show the start of a negative bay (lowest panel of Fig. 23).

When the SOSMAG magnetic field reaches its minimum, the MMS plasma instrument shows the start of a strong tailward flow, $V x \approx-500 \mathrm{~km} / \mathrm{s}$, which changes to a strong Earthward flow, $V x \approx 500 \mathrm{~km} / \mathrm{s}$, indicating that the MMS spacecraft were crossed by the tailward moving X-line. The SOSMAG field, however, has already recovered before the Earthward flow measured by MMS.

The measurements by SOSMAG at geostationary location are important for this kind of investigation because they show the response of the inner magnetosphere to the flow burst that was observed in the tail. For example, the decrease in total magnetic field is either related to the stretching of the tail, "pulling out the inner magnetosphere field" during the growth phase, which then returns with a "bang" when reconnection starts. This can probably also be described by the set-up of a current system, the substorm current wedge.

Space weather at Earth is largely driven by magnetic reconnection at the dayside magnetopause and, consequently, in the geomagnetic tail. However, magnetic reconnection is not the only reason for geomagnetic variations. Solar wind pressure pulses or periodic variations may set off magnetospheric Ultra-Low Frequency (ULF) waves (e.g. Sibeck et al. 1989), so may IMF rotations (e.g. Farrugia et al. 2008). The bow shock and the region upstream of it that is magnetically connected to the shock, the foreshock, are also sources of phenomena that have been associated with ULF wave activity in the magnetosphere (Eastwood et al. 2011). These phenomena include hot flow anomalies, foreshock bubbles, or compressional foreshock waves (e.g. Sibeck et al. 1999; Turner et al. 2013). For the foreshock to have a major influence on the magnetosphere, it should be located in the subsolar region upstream of the shock. Therefore, IMF cone angle should be low; that is the angle between the Earth-Sun-line and the IMF direction. Under these low cone angle conditions, magnetosheath jets are oftentimes observed inside the magnetosheath (e.g. Plaschke et al. 2018). They are generated at the bow shock and are able to propagate through the entire magnetosheath, thereby impacting on the magnetopause. There, they are able to set off (standing) magnetopause surface waves and/or ULF waves inside the magnetosphere (e.g. Archer et al. 2019). In particular under northward IMF and fast solar wind conditions, the Kelvin-Helmholtz-instability developing along the flanks of the magnetopause is also known to be a major source of ULF wave activity inside the magnetosphere (e.g. Kavosi and Raeder 2015).

SOSMAG aboard GK-2A is able to measure and clearly resolve even small-scale ULF wave activity on geostationary orbit. This can be seen in Fig. 25, which displays the SOSMAG IMF data for 11 May 2019 in the dayside noon and afternoon local time sectors. The ULF fluctuations can be discerned already in the Hpen time series (top panel). After subtracting a 10 minute moving average, they are more clearly visible in the second panel. The 


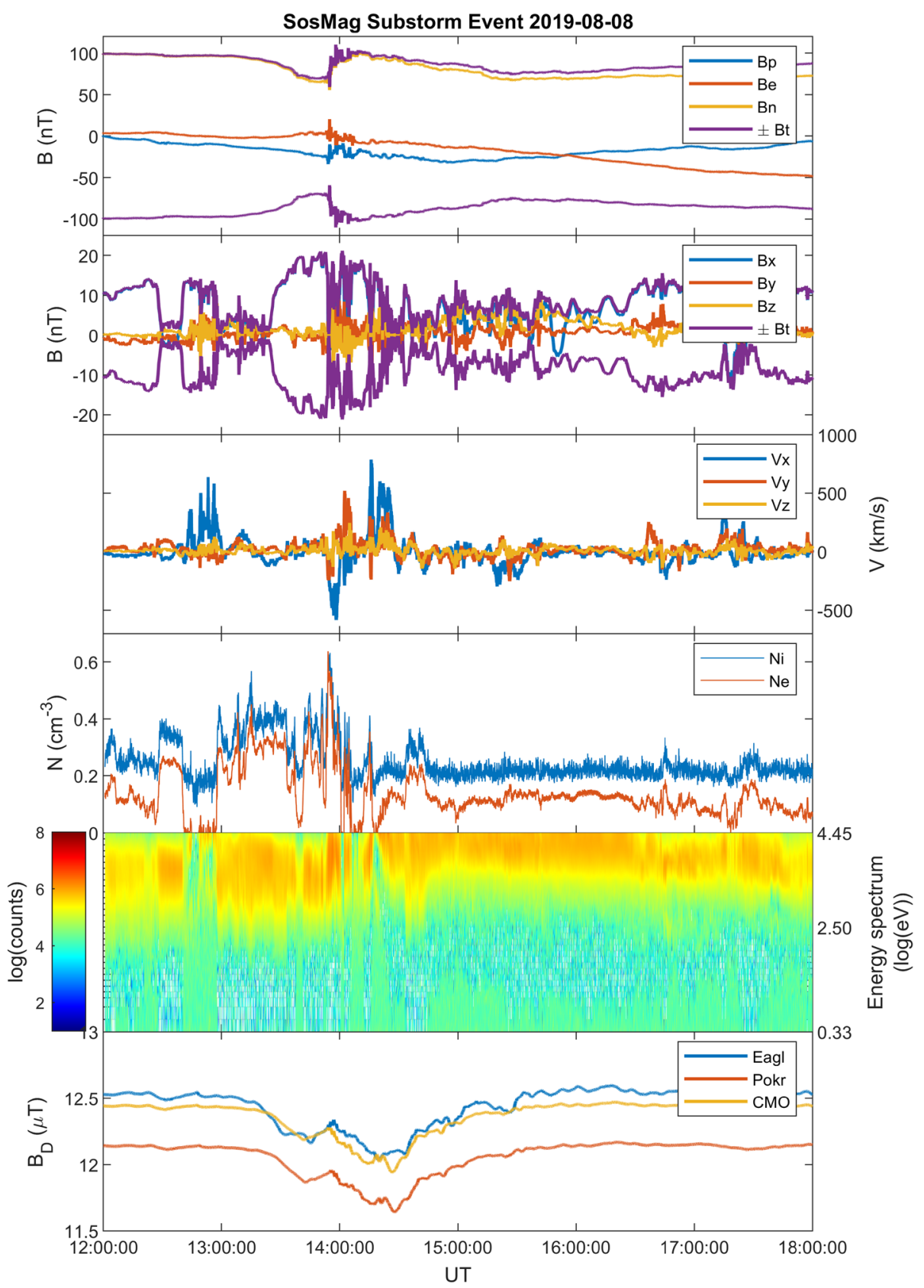

Fig. 23 Comparison of SOSMAG (top panel), MMS (four middle panels) and ground based data (lowest panel) during a substorm event on 8 August 2019

first wave packet between 2:20 and 4:20 UT occurs during southward IMF conditions (see GSM Z in third panel), and may therefore be associated to reconnection or to resulting flux transfer events (e.g. Lockwood and Wild 1993). Such an explanation would, however, raise 


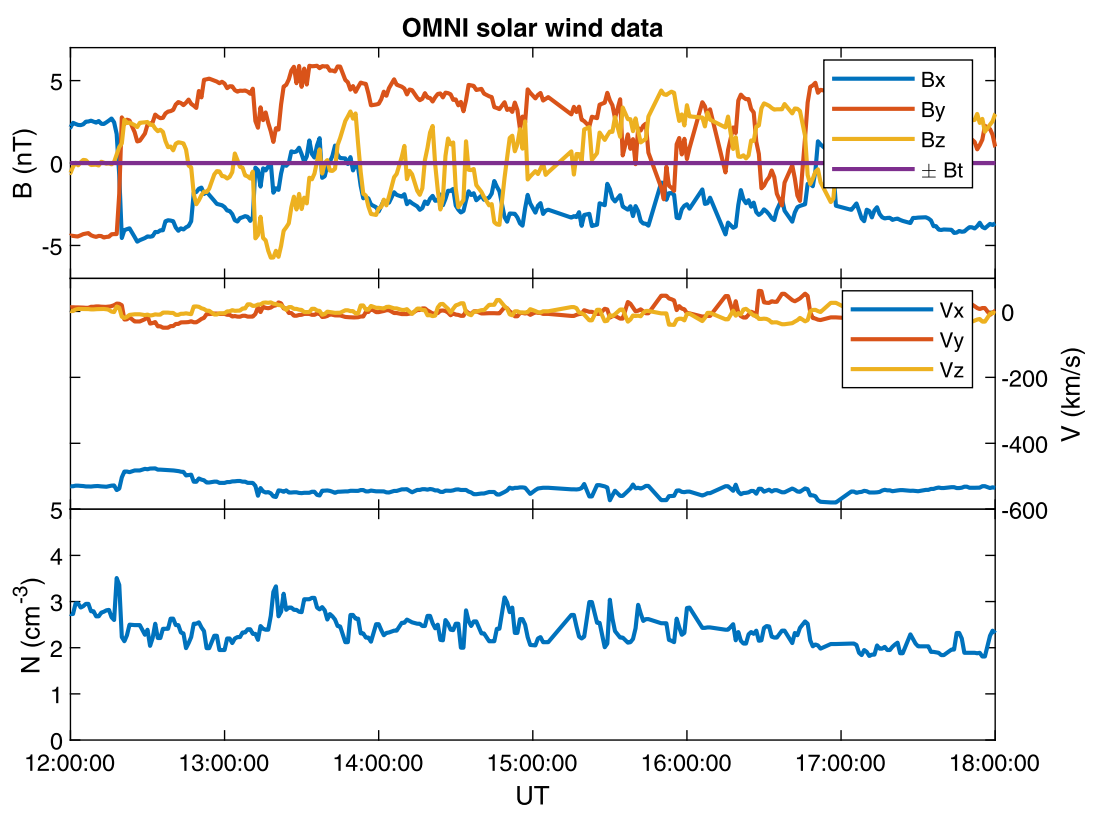

Fig. 24 Solar wind data processed for the nose of the magnetopause on 8 August 2019 (data from the OMNIWeb of NASA; https://omniweb.gsfc.nasa.gov/)

the question of why the wave activity did not start earlier when the IMF was pointing even more in southward direction. The second wave packet, starting approximately at 5:00 UT, is initiated however during northward IMF at the flanks. There, Kelvin-Helmholtz-instability is known to be a major source of wave activity, but in this case the solar wind velocity (bottom panel) is only about $360 \mathrm{~km} / \mathrm{s}$ and, therefore, very low. Foreshock phenomena or magnetosheath jets are unlikely to be the cause of the observed wave activity as the IMF cone angle was consistently large during the entire time interval of interest (fourth panel). This example shows that the reason for ULF wave activity may not always be straightforwardly identifiable, justifying the need for future scientific research and a larger density of in-orbit measurement points.

\section{Data Product Flow}

SOSMAG data are transmitted from GK-2A to the South Korean ground station in real-time and first stored in the GK-2A ground system managed by the National Meteorological Satellite Center of the Korea Meteorological Administration (KMA). From there, the data are retrieved by the SOSMAG Ground Processor (GP), which is installed at the Space Weather Service Centre of ESA, every minute.

Data within the GP is processed according to the flow presented in Fig. 26. Data which are retrieved from KMA are used to process both, on-board cleaned and raw data (dependent on availability) from binary data to magnetic field data in $\mathrm{nT}$. The presented processing flow is executed three times with different delays as well as different parameter qualities. The properties of the resulting data sets are summarized in Table 7. Real-time and preliminary data use preliminary calibration and cleaning parameters from a previous determination 


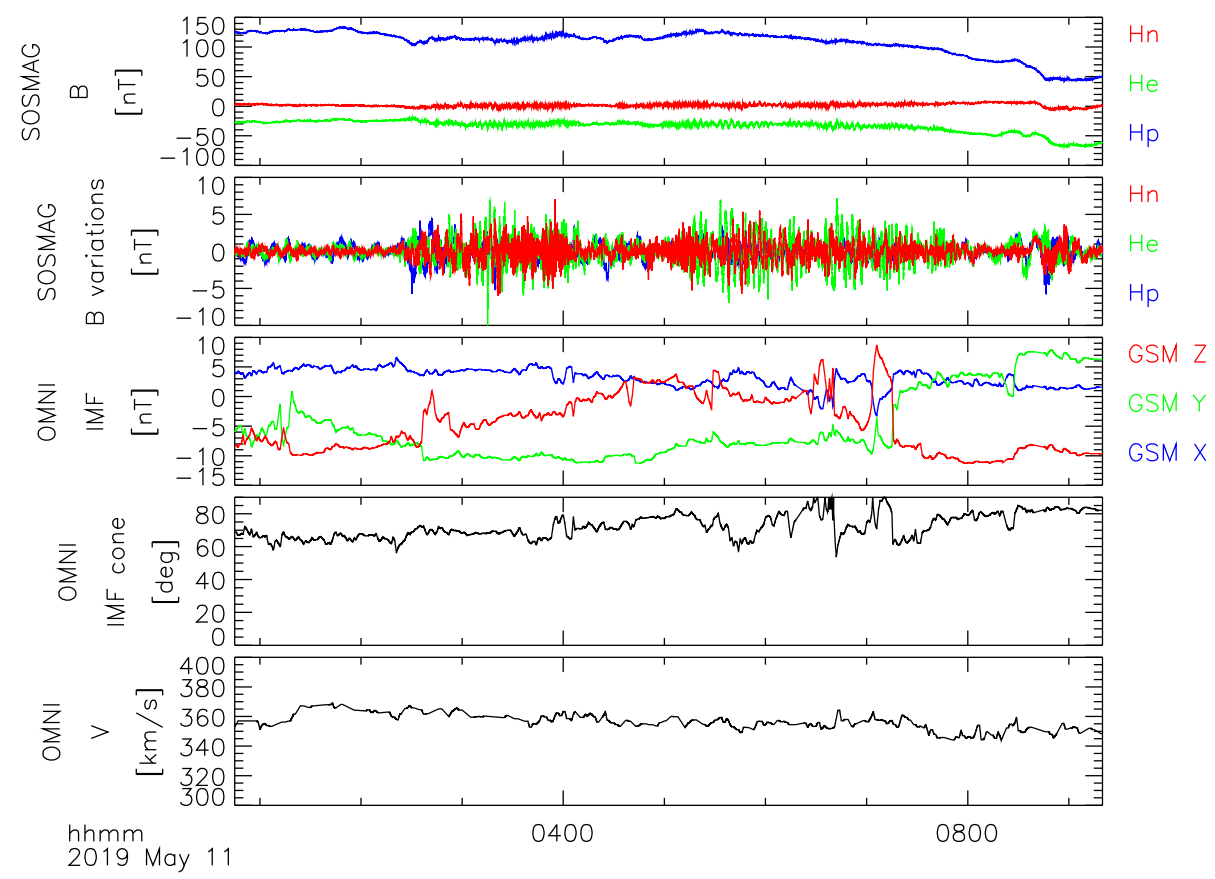

Fig. 25 From top to bottom with time in UT: GK-2A SOSMAG magnetic field in Hpen, top panel measurements from which 10 minute averages have been subtracted, NASA OMNIWeb high resolution IMF measurements in Geocentric Solar Magnetospheric coordinate system, IMF cone angle and solar wind proton velocity

to provide data as early and complete as possible. Final data are processed using updated parameters, which were generated with the procedures described in Sects. 5.2 and 5.3. In addition, periods with higher distortion levels are flagged to indicate those data which have lower accuracy.

The resulting three data sets are available as level 2 science data products in Hpen and GSE coordinate systems and are complemented with satellite attitude information. From October 2020, the processed data will be made available within the frame of ESA's Space Weather Portal (http://swe.ssa.esa.int/) which is open to registered public users free of charge. A Representational State Transfer (REST) Application Programming Interface (API) like the Heliophysics Application Programming Interface (HAPI) managed by NASA is currently in discussion, but not yet decided.

In parallel to the provision of the data via the Space Weather Portal, which mainly supports the space weather application driven activities, it is planned to make the final data (Table 7) available in Common Data Format from a server at the Space Research Institute of the Austrian Academy of Sciences.

\section{Summary and Conclusions}

The Service Oriented Spacecraft Magnetometer (SOSMAG) is equipped with four magnetic field sensors whereas two sensors are mounted on an approximately one meter long boom, which was developed as part of the instrument. Aboard the South Korean meteorological 
Fig. 26 Data flow within the SOSMAG ground processor

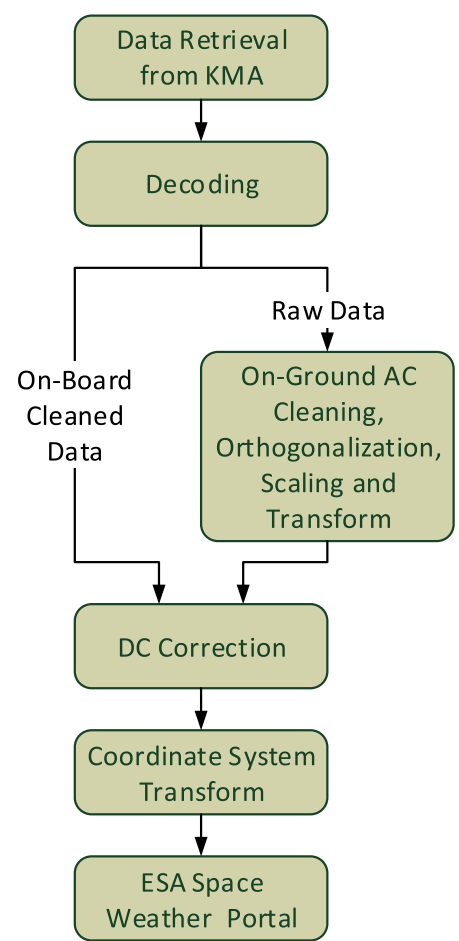

Table 7 Output data types of the SOSMAG ground processor

\begin{tabular}{lll}
\hline Data type & Generation delay & Description \\
\hline Real-time & $<5$ minutes & $\begin{array}{l}\text { Real-time data are generated almost immediately, but uses cleaning and } \\
\text { calibration parameters which were determined with data which were } \\
\text { measured up to } 98 \text { days before. }\end{array}$ \\
Preliminary & $1-2$ days & $\begin{array}{l}\text { Preliminary data are generated in the same way as real-time data, but they } \\
\text { include data that might have arrived late, e.g. due to service outages. }\end{array}$ \\
Final & 98 days & $\begin{array}{l}\text { Final data are processed using the newest parameter sets that were generated } \\
\text { specifically for these data. In addition, data quality flags are added. }\end{array}$ \\
\hline
\end{tabular}

and environmental satellite GEO-KOMPSAT-2A (GK-2A), SOSMAG was launched into a geostationary orbit at a geographic longitude of $128.2^{\circ}$ East as part of the Korea Space Environment Monitor instrument suite on 4 December 2018. The nominal operation of SOSMAG started on 25 February 2019.

The hosting spacecraft was designed and built without magnetic cleanliness requirements, and the location of the boom and of the two magnetic sensors within the spacecraft was chosen without magnetic cleanliness related considerations. Furthermore, the SOSMAG team had no access to operation time tables and housekeeping data of the spacecraft during the first 12 months of operation.

The combination of multiple sensors with at least two sensors on a short boom was chosen to achieve an accuracy level which is good enough for space weather related forecast and science under conditions which are not ideal for spaceborne magnetic measurements. 
It has been the goal to derive the correction and calibration parameters during the first months of operation with post-processing on ground so that, after an upload of the parameters to the spacecraft, SOSMAG is able to process corrected and cleaned magnetic vectors on board. In an ideal case, this should allow for a change of the data transmission mode from the raw data of the four sensors with one vector per second each to a cleaned data product from a virtually single sensor with four vectors per second.

The cleaning of the SOSMAG data from dynamic disturbances generated by the GK2A spacecraft is done with a newly developed Principal Component Gradiometer (PiCoG) method which employs principal component analysis to decouple multiple disturbance sources and to minimize the introduction of artefacts to the components free of the targeted disturbance. The magnitudes of the identified disturbances, which can be as large as $40 \mathrm{nT}$ in the sensor furthest away from the spacecraft, are reduced by a minimum factor of 2 and a maximum factor of 35, depending on the location and the signature of the disturbance. The magnitude of the largest remnant field in the cleaned data from a spacecraft disturber is $2.0 \mathrm{nT}$. A better reduction is hindered by the quite high intrinsic noise as well as by the non-ideal mounting location of the two AMR based spacecraft sensors.

A magnetic screening campaign has been conducted at KARI facilities pre-flight. The magnetic signatures of the reaction wheels and the Advance Meteorological Imager (AMI), which is the primary payload of GK-2A, have been investigated by the SOSMAG sensors and two fluxgate based gradiometers which were placed nearby the spacecraft. While the amplitude of the magnetic stray field of the reaction wheels was low, recurrent spikes of the AMI (about $10 \mathrm{nT}$ peak at the inboard fluxgate sensor) could be detected. Both, the low level of reaction wheel interference and the peak values of the AMI generated spikes, are confirmed by the inflight measurements. These interferences can be eliminated by the multisensor arrangement as expected and even though not all potential spacecraft disturbers could be switched on during this magnetic screening campaign on ground, it was an important first step for validating the SOSMAG concept.

Due to a daily shadowing of the SOSMAG boom, the temperature of the outboard sensor cycles every day between $+30^{\circ} \mathrm{C}$ and $-90{ }^{\circ} \mathrm{C}$. This causes a specific dynamic disturbance, which cannot be corrected using the PiCoG method. Since the sensor has not been designed for extreme temperature changes, sensor intrinsic offset oscillations with a periodicity up to 60 minutes and peak-to-peak values up to $5 \mathrm{nT}$ are generated and still present in the corrected data product especially during the cooling phase.

Taking the currently remaining artefacts into account, the error requirement of less than $1 \mathrm{nT}$ for frequencies higher than $0.1 \mathrm{mHz}$ has not been met yet but the leftovers are well characterized and will be flagged accordingly in the final data product.

The low frequency error of the corrected SOSMAG data for signal frequencies of less than $0.1 \mathrm{mHz}$ (i.e. the offset) was evaluated by verifying the SOSMAG data against the Tsyganenko Earth's field model on a daily and a long-term basis with a 28 days long sliding window, as well as by comparison with the magnetic field measurements aboard the foursatellite Magnetospheric Multiscale mission. It was demonstrated that the offset is below the required $5 \mathrm{nT}$. Furthermore, the drift of the offsets over 10 months is less than $7 \mathrm{nT}$.

The discussion of two science phenomena observed by SOSMAG, a substorm event and ultra-low frequency waves, clearly demonstrate the suitability of the SOSMAG data for space weather forecast applications in particular and magnetospheric science in general. This is of specific importance because the magnetic field measurements aboard GK-2A complement the measurements of the geostationary GOES satellites in a completely different longitudinal sector.

Future work could include a further reduction of the remaining artefacts in the final data product with a focus on lessening the temperature driven sensor oscillations with an epoch 
based identification and correction of the offset drift. While this work is ongoing, the transmission of the raw data from all four sensors with one vector per second is maintained and the mode change to a virtually single sensor with four vectors per second is envisaged for a later point in time.

Acknowledgements The development and test of the SOSMAG flight model including calibration on ground, in-orbit verification and in-flight calibration has been funded by the Space Safety (formerly Space Situational Awareness) Programme of the European Space Agency (project no. 4000117456). The preceding development and test of the engineering and qualification model was funded by the General Support Technology Programme of ESA (project no. 4000105630). Both optional programmes were accordingly and gratefully supported by the national space agencies of the participating countries: German Aerospace Center, Austrian Aeronautics and Space Agency and UK Space Agency.

We acknowledge the use of magnetometer data from the GOES satellites, Level 2 fluxgate magnetometer and fast plasma investigation data from the Magnetospheric Multiscale (MMS) mission, solar wind data from the OMNI webpage of NASA as well as ground based measurements from the THEMIS website. The MMS and OMNIWeb data are stored at https://lasp.colorado.edu/mms/sdc/ and https://omniweb.gsfc.nasa.gov/, respectively. The GOES data can be downloaded from the NOAA Space Weather Prediction Center at http://www.swpc.noaa.gov/ and the ground based data were downloaded from http://themis.ssl.berkeley.edu/index.shtml. All data sets are publicly available.

Publisher's Note Springer Nature remains neutral with regard to jurisdictional claims in published maps and institutional affiliations.

Open Access This article is licensed under a Creative Commons Attribution 4.0 International License, which permits use, sharing, adaptation, distribution and reproduction in any medium or format, as long as you give appropriate credit to the original author(s) and the source, provide a link to the Creative Commons licence, and indicate if changes were made. The images or other third party material in this article are included in the article's Creative Commons licence, unless indicated otherwise in a credit line to the material. If material is not included in the article's Creative Commons licence and your intended use is not permitted by statutory regulation or exceeds the permitted use, you will need to obtain permission directly from the copyright holder. To view a copy of this licence, visit http://creativecommons.org/licenses/by/4.0/.

\section{References}

L.N.S. Alconcel, P. Fox, P. Brown, T.M. Oddy, E.L. Lucek, C.M. Carr, Geosci. Instrum. Method. Data Syst. 3, 95 (2014)

V. Angelopoulos, A. Artemyev, T.D. Phan, Y. Miyashita, Nat. Phys. 1 (2020)

M.O. Archer, H. Hietala, M.D. Hartinger, F. Plaschke, V. Angelopoulos, Nat. Commun. 10, 1 (2019)

H.U. Auster, A. Lichopoj, J. Rustenbach, H. Bitterlich, K.H. Fornacon, O. Hillenmaier, R. Krause, H.J. Schenk, V. Auster, Meas. Sci. Technol. 6, 477 (1995)

H.U. Auster, I. Apathy, G. Berghofer, A. Remizov, R. Roll, K.H. Fornacon, K.H. Glassmeier, G. Haerendel, I. Hejja, E. Kührt, W. Magnes, D. Moehlmann, U. Motschmann, I. Richter, H. Rosenbauer, C.T. Russell, J. Rustenbach, K. Sauer, K. Schwingenschuh, I. Szemerey, R. Waesch, Space Sci. Rev. 128, 221 (2007)

H.U. Auster, K.H. Glassmeier, W. Magnes, O. Aydogar, W. Baumjohann, D. Constantinescu, D. Fischer, K.H. Fornacon, E. Georgescu, P. Harvey, O. Hillenmaier, R. Kroth, M. Ludlam, Y. Narita, R. Nakamura, K. Okrafka, F. Plaschke, I. Richter, H. Schwarzl, B. Stoll, A. Valavanoglou, M. Wiedemann, Space Sci. Rev. 141, 235 (2008)

A. Balogh, Space Sci. Rev. 152, 23 (2010)

J.-P. Bibring, H. Rosenbauer, H. Boehnhardt, S. Ulamec, J. Biele, S. Espinasse, B. Feuerbacher, P. Gaudon, P. Hemmerich, P. Kletzkine, D. Moura, R. Mugnuolo, G. Nietner, B. Pätz, R. Roll, H. Scheuerle, K. Szegö, K. Wittmann (Philae Project Office and The Entire Philae Team), Space Sci. Rev. 128, 205 (2007)

J.E. Borovsky, M.H. Denton, J. Geophys. Res. 115, A08217 (2010)

P. Brown, T. Beek, C. Carr, H. O’Brien, E. Cupido, T. Oddy, T.S. Horbury, Meas. Sci. Technol. 23, 025902 (2012)

J.L. Burch, T.E. Moore, R.B. Torbert, B.L. Giles, Space Sci. Rev. 199, 5 (2016)

O.D. Constantinescu, H.-U. Auster, M. Delva, O. Hillenmaier, W. Magnes, F. Plaschke, Geosci. Instrum. Method. Data Syst. (2020). https://doi.org/10.5194/gi-2020-10 
J.P. Eastwood, S.J. Schwartz, T.S. Horbury, C.M. Carr, K.-H. Glassmeier, I. Richter, C. Koenders, F. Plaschke, J.A. Wild, J. Geophys. Res. 116 (2011)

J.P. Eastwood, E. Biffis, M.A. Hapgood, L. Green, M.M. Bisi, R.D. Bentley, R. Wicks, L.-A. McKinnell, M. Gibbs, C. Burnett, Risk Anal. 37, 206 (2017a)

J.P. Eastwood, R. Nakamura, L. Turc, L. Mejnertsen, M. Hesse, Space Sci. Rev. 212, 1221 (2017b)

C.J. Farrugia, F.T. Gratton, E.J. Lund, P.E. Sandholt, S.W.H. Cowley, R.B. Torbert, G. Gnavi, I.R. Mann, L. Bilbao, C. Mouikis, L. Kistler, C.W. Smith, H.J. Singer, J.F. Watermann, J. Geophys. Res. 113 (2008)

K.-H. Fornacon, H.U. Auster, E. Georgescu, W. Baumjohann, K.-H. Glassmeier, G. Haerendel, J. Rustenbach, M. Dunlop, Ann. Geophys. 17, 1521 (1999)

K.-H. Glassmeier, I. Richter, A. Diedrich, G. Musmann, U. Auster, U. Motschmann, A. Balogh, C. Carr, E. Cupido, A. Coates, M. Rother, K. Schwingenschuh, K. Szegö, B. Tsurutani, Space Sci. Rev. 128, 649 (2007)

M. Hapgood, Nature 484, 311 (2012)

S. Kavosi, J. Raeder, Nat. Commun. 6, 1 (2015)

D.J. Knipp, Understanding Space Weather and the Physics Behind It (McGraw-Hill Education, New York, 2011)

S. Leitner, A. Valavanoglou, P. Brown, C. Hagen, W. Magnes, B.J. Whiteside, C.M. Carr, M. Delva, W. Baumjohann, IEEE Trans. Magn. 51, 1 (2015)

J. Lenz, S. Edelstein, IEEE Sens. J. 6, 631 (2006)

R.P. Lepping, M.H. Acũna, L.F. Burlaga, W.M. Farrell, J.A. Slavin, K.H. Schatten, F. Mariani, N.F. Ness, F.M. Neubauer, Y.C. Whang, J.B. Byrnes, R.S. Kennon, P.V. Panetta, J. Scheifele, E.M. Worley, Space Sci. Rev. 71, 207 (1995)

M. Lockwood, M.N. Wild, J. Geophys. Res. 98, 5935 (1993)

T.M. Loto'aniu, R.J. Redmon, S. Califf, H.J. Singer, W. Rowland, S. Macintyre, C. Chastain, R. Dence, R. Bailey, E. Shoemaker, F.J. Rich, D. Chu, D. Early, J. Kronenwetter, M. Todirita, Space Sci. Rev. 215, 32 (2019)

W. Magnes, M. Oberst, A. Valavanoglou, H. Hauer, C. Hagen, I. Jernej, H. Neubauer, W. Baumjohann, D. Pierce, J. Means, P. Falkner, Meas. Sci. Technol. 19, 115801 (2008)

M.B. Moldwin, An Introduction to Space Weather, 1st edn. (Cambridge University Press, Cambridge, 2008)

N.F. Ness, K.W. Behannon, R.P. Lepping, K.H. Schatten, J. Geophys. Res. 76, 3564 (1971)

F. Plaschke, Geosci. Instrum. Method. Data Syst. 8, 285 (2019)

F. Plaschke, H. Hietala, M. Archer, X. Blanco-Cano, P. Kajdič, T. Karlsson, S.H. Lee, N. Omidi, M. Palmroth, V. Roytershteyn, D. Schmid, V. Sergeev, D. Sibeck, Space Sci. Rev. 214, 81 (2018)

F. Plaschke, H.-U. Auster, D. Fischer, K.-H. Fornaçon, W. Magnes, I. Richter, D. Constantinescu, Y. Narita, Geosci. Instrum. Method. Data Syst. 8, 63 (2019)

F. Primdahl, J. Phys. E, Sci. Instrum. 12, 241 (1979)

T.I. Pulkkinen, N.Y. Ganushkina, E.I. Tanskanen, M. Kubyshkina, G.D. Reeves, M.F. Thomsen, C.T. Russell, H.J. Singer, J.A. Slavin, J. Gjerloev, J. Geophys. Res. 111, A11S17 (2006)

C.T. Russell, B.J. Anderson, W. Baumjohann, K.R. Bromund, D. Dearborn, D. Fischer, G. Le, H.K. Leinweber, D. Leneman, W. Magnes, J.D. Means, M.B. Moldwin, R. Nakamura, D. Pierce, F. Plaschke, K.M. Rowe, J.A. Slavin, R.J. Strangeway, R. Torbert, C. Hagen, I. Jernej, A. Valavanoglou, I. Richter, Space Sci. Rev. 199, 189 (2016)

J. Seon, K.-S. Chae, G.W. Na, H.-K. Seo, Y.-C. Shin, J. Woo, C.-H. Lee, W.-H. Seol, C.-A. Lee, S. Pak, H. Lee, S.-H. Shin, D.E. Larson, K. Hatch, G.K. Parks, J. Sample, M. McCarthy, C. Tindall, Y.-J. Jeon, J.-K. Choi, J.-Y. Park, Space Sci. Rev. 216, 13 (2020)

D.G. Sibeck, W. Baumjohann, R.C. Elphic, D.H. Fairfield, J.F. Fennell, W.B. Gail, L.J. Lanzerotti, R.E. Lopez, H. Luehr, A.T.Y. Lui, C.G. Maclennan, R.W. McEntire, T.A. Potemra, T.J. Rosenberg, K. Takahashi, J. Geophys. Res. 94, 2505 (1989)

D.G. Sibeck, N.L. Borodkova, S.J. Schwartz, C.J. Owen, R. Kessel, S. Kokubun, R.P. Lepping, R. Lin, K. Liou, H. Lühr, R.W. McEntire, C.-I. Meng, T. Mukai, Z. Nemecek, G. Parks, T.D. Phan, S.A. Romanov, J. Safrankova, J.-A. Sauvaud, H.J. Singer, S.I. Solovyev, A. Szabo, K. Takahashi, D.J. Williams, K. Yumoto, G.N. Zastenker, J. Geophys. Res. 104, 4577 (1999)

H. Singer, L. Matheson, R. Grubb, A. Newman, D. Bouwer, GOES-8 and Beyond E.R. Washwell Proc. SPIE, vol. 2812 (Int. Soc. for Opt. Eng., Bellingham, 1996), pp. 299-308

N.A. Stutzke, S.E. Russek, D.P. Pappas, M. Tondra, J. Appl. Phys. 97, 10 Q107 (2005)

R.B. Torbert, C.T. Russell, W. Magnes, R.E. Ergun, P.-A. Lindqvist, O. LeContel, H. Vaith, J. Macri, S. Myers, D. Rau, J. Needell, B. King, M. Granoff, M. Chutter, I. Dors, G. Olsson, Y.V. Khotyaintsev, A. Eriksson, C.A. Kletzing, S. Bounds, B. Anderson, W. Baumjohann, M. Steller, K. Bromund, G. Le, R. Nakamura, R.J. Strangeway, H.K. Leinweber, S. Tucker, J. Westfall, D. Fischer, F. Plaschke, J. Porter, K. Lappalainen, Space Sci. Rev. 199, 105 (2016a) 
R.B. Torbert, H. Vaith, M. Granoff, M. Widholm, J.A. Gaidos, B.H. Briggs, I.G. Dors, M.W. Chutter, J. Macri, M. Argall, D. Bodet, J. Needell, M.B. Steller, W. Baumjohann, R. Nakamura, F. Plaschke, H. Ottacher, J. Hasiba, K. Hofmann, C.A. Kletzing, S.R. Bounds, R.T. Dvorsky, K. Sigsbee, V. Kooi, Space Sci. Rev. 199, 283 (2016b)

N.A. Tsyganenko, Planet. Space Sci. 37, 5 (1989)

N.A. Tsyganenko, M.I. Sitnov, J. Geophys. Res. Space Phys. 110 (2005)

D.L. Turner, V. Angelopoulos, W. Li, M.D. Hartinger, M. Usanova, I.R. Mann, J. Bortnik, Y. Shprits, J. Geophys. Res. Space Phys. 118, 2196 (2013)

T.L. Zhang, W. Baumjohann, M. Delva, H.-U. Auster, A. Balogh, C.T. Russell, S. Barabash, M. Balikhin, G. Berghofer, H.K. Biernat, H. Lammer, H. Lichtenegger, W. Magnes, R. Nakamura, T. Penz, K. Schwingenschuh, Z. Vörös, W. Zambelli, K.-H. Fornacon, K.-H. Glassmeier, I. Richter, C. Carr, K. Kudela, J.K. Shi, H. Zhao, U. Motschmann, J.-P. Lebreton, Planet. Space Sci. 54, 1336 (2006) 